\title{
Yves Le Grand on matrices in optics with applica- tion to vision: Translation and critical analysis
}

\author{
William Frith Harris
}

Department of Optometry, University of Johannesburg, P O Box 524, Auckland Park 2006, South Africa

<wharris@uj.ac.za>

Received 10 July 2013; revised version accepted 4 December 2013

\begin{abstract}
An appendix to Le Grand's 1945 book, Optique Physiologique: Tome Premier: La Dioptrique de l'Eil et Sa Correction, briefly dealt with the application of matrices in optics. However the appendix was omitted from the well-known English translation, Physiological Optics, which appeared in 1980. Consequently the material is all but forgotten. This is unfortunate in view of the importance of the dioptric power matrix and the ray transference which entered the optometric literature many years later. Motivated by the perception that there has not been enough care in optometry to attribute concepts appropriately this paper attempts a careful analysis of Le Grand's thinking as reflected in his appendix. A translation into English is provided in the appendix to this
\end{abstract}

paper. The paper opens with a summary of the basics of Gaussian and linear optics sufficient for the interpretation of Le Grand's appendix which follows. The paper looks more particularly at what Le Grand says in relation to the transference and the dioptric power matrix though many other issues are also touched on including the conditions under which distant objects will map to clear images on the retina and, more particularly, to clear images that are undistorted. Detailed annotations of Le Grand's translated appendix are provided. (S Afr Optom 2013 72(4) 145-166)

Key words: Le Grand, Gaussian optics, linear optics, dioptric power matrix, ray transference, condition for clear retinal images, condition for undistorted images, astigmatism

\section{Introduction}

Matrix methods have played significant roles in modern ophthalmic optics. ${ }^{1-23}$ In particular the dioptric power matrix was introduced into optometry by Long $^{20}$ in 1976 and the system matrix or ray transference by Keating ${ }^{22,23}$ in 1981. As pointed out by Blendowske ${ }^{24}$, however, much of the material had actually been published before by Fick in the early 1970s in a series of 22 short articles; one in particular describes the dioptric power matrix ${ }^{25}$.
Because he 'published in German and additionally in a journal more related to the craftsman than to a scientific readership [Fick's] approach was nearly forgotten' ${ }^{24}$ Indeed there is earlier material still which unfortunately seems to have has suffered the same fate, also, apparently, for not having been published in English: an appendix entitled 'Le Calcul des Matrices en Optique' (pages 322-328) to the book Optique Physiologique: Tome Premier: la Dioptrique de l'Eil et $\mathrm{Sa}$ Correction published by Le Grand ${ }^{26}$ in 1945. The 3rd edition of the book ${ }^{27}$ (dated 1964), with 
minor changes to the appendix, appeared in English translation $^{28}$ in 1980 as the well-known Physiological Optics by Le Grand and El Hage ${ }^{29}$. Sadly, however, the appendix was omitted from the English version.

Le Grand's appendix is interesting in its own right. However it is not easy to come by, is terse, is in French, gives few explanations and uses symbolism that differs from that in current use. For these reasons it seems appropriate to make it available in English translation for the modern vision scientist. Our purpose here, then, is to do so and to provide a critical analysis of the appendix set in the context of matrix methods in current use in optometry and vision science.

We begin with the basics of Gaussian and linear optics expressed in the matrix symbolism in recent usage. With that as reference we then examine Le Grand's appendix. His appendix is given in English translation in the appendix to this paper together with detailed annotations.

\section{Elements of matrix methods in Gaussian and linear optics}

Gaussian and linear optics are both first-order optical models. The first is effectively a twodimensional optics (Figure 1). Rays can be visualized in a single plane, the plane containing the optical axis and the object and image points; usually it is a representative plane in systems invariant under rotation about the optical axis. The second model is a three-dimensional generalization. Only the latter is adequately capable of handling astigmatism, a fundamentally three-dimensional phenomenon. For an excellent account of both models of optics, and their relationship to geometrical and other models, the reader is referred elsewhere ${ }^{30}$. We summarize here basic results which we shall need in our analysis of Le Grand's appendix below.

\section{The transference}

The matrix of interest goes by several names including system matrix ${ }^{22,23,31-33}$, matrix of the system ${ }^{34,35}$, lens matrix $^{36}, A B C D$ matrix ${ }^{37}$, optical matrix ${ }^{37}$, ray-transfer matrix $^{37,38}$ and (ray) transference ${ }^{15-18}$. We shall refer to it here as the transference and represent it by the symbol S. It is a complete representation of the first-order optical character of a system. ${ }^{37}$
In Gaussian optics the transference is a real $2 \times 2$ matrix which we write $\mathrm{as}^{30,38}$

$\mathbf{S}=\left(\begin{array}{ll}A & B \\ C & D\end{array}\right)$

where $A, B, C$ and $D$ are what we call the four fundamental (first-order optical) properties of Gaussian system S. ${ }^{39-41} A$ is the dilation, $B$ the disjugacy, $C$ the divergence and $D$ the divarication. The four fundamental properties are not independent but are related by the equation

$A D-C B=1$.

In other words the transference has a unit determinant ${ }^{30,38}$. We say that the $2 \times 2$ transference has three degrees of freedom. If we know three of the fundamental properties we can usually calculate the remaining one. We cannot always do so, however; for example we cannot calculate $D$ if we know $A, B$ and $C$ and $A$ happens to be 0 .

In linear optics the transference expands to become a $4 \times 4$ real matrix which is usually written ${ }^{9,37}$

$\mathbf{S}=\left(\begin{array}{ll}\mathbf{A} & \mathbf{B} \\ \mathbf{C} & \mathbf{D}\end{array}\right)$

in terms of four $2 \times 2$ submatrices $\mathbf{A}, \mathbf{B}, \mathbf{C}$ and $\mathbf{D}$, the four fundamental properties of the linear system. Other properties can be obtained from the fundamental properties; they are derived properties. For example the dioptric power of a system is defined by ${ }^{39}$

$\mathbf{F}=-\mathbf{C}$.

The $4 \times 4$ transference has 10 degrees of freedom, the 16 entries being related by six scalar equations instead of just one (Equation 2). The six equations are contained within the single matrix equation ${ }^{30,37,42-48}$

$\mathbf{S}^{\mathrm{T}} \mathbf{E S}=\mathbf{E}$

where $\mathbf{S}^{\mathrm{T}}$ represents the matrix transpose of $\mathbf{S}$,

$\mathbf{E}=\left(\begin{array}{cc}\mathbf{O} & \mathbf{I} \\ -\mathbf{I} & \mathbf{O}\end{array}\right)$

and $\mathbf{O}$ and $\mathbf{I}$ are $2 \times 2$ null and identity matrices respectively. Substitution from Equations 3 and 6 into Equation 5 leads to three matrix equations, ${ }^{43,48,49}$

$$
\begin{aligned}
& \mathbf{A}^{\mathrm{T}} \mathbf{C}=\mathbf{C}^{\mathrm{T}} \mathbf{A}, \\
& \mathbf{B}^{\mathrm{T}} \mathbf{D}=\mathbf{D}^{\mathrm{T}} \mathbf{B},
\end{aligned}
$$




$$
\mathbf{A}^{\mathrm{T}} \mathbf{D}-\mathbf{C}^{\mathrm{T}} \mathbf{B}=\mathbf{I}
$$

If we multiply these equations out in terms of their entries we find that Equations 7 and 8 are each equivalent to a single scalar equation and Equation 9 is equivalent to four scalar equations.

The $2 \times 2$ transference (Equation 1) also obeys Equation 5 if $\mathbf{O}$ and $\mathbf{I}$ are interpreted as $1 \times 1$ null and identity matrices, that is, simply as the scalars 0 and 1. It also obeys Equations 7 and 8 but trivially and Equations 2 and 9 are identical.

Any $2 n \times 2 n$ matrix $\mathbf{S}$ which obeys Equation 5, where $\mathbf{O}$ and $\mathbf{I}$ are $n \times n$, is said to be symplectic ${ }^{42-46}$. In particular both $2 \times 2$ and $4 \times 4$ transferences are symplectic. Many results for transferences arising as a consequence of their symplecticity are summarized elsewhere ${ }^{47}$; some will be used below.

Like Le Grand ${ }^{26,27}$ we shall be concerned here with optical systems all of whose component refracting elements are centred on a common axis, the optical axis. For systems with decentred elements one can make use of uncentered ${ }^{49}$ or augmented symplectic matrices ${ }^{50-53}$ which have an extra row and an extra column.

\section{A ray traversing a system}

Figure 1 represents a ray traversing a Gaussian system $\mathrm{S}$. $\mathrm{S}$ lies between entrance and exit planes $\mathrm{T}_{0}$ and $\mathrm{T}$. The elements of $\mathrm{S}$ (none of which is shown) are centred on optical axis $\mathrm{Z}$. Upstream of $\mathrm{S}$ the medium has index of refraction $n_{0}$; downstream from $\mathrm{S}$ the index is $n$. Transverse positions and inclinations are measured relative to $\mathrm{Z}$. At incidence the ray has state $^{30,37}$

$\boldsymbol{\rho}_{0}=\left(\begin{array}{l}y_{0} \\ \alpha_{0}\end{array}\right)$,

a $2 \times 2$ matrix, where $y_{0}$ is the transverse position,

$$
\alpha_{0}=n_{0} a_{0}
$$

and $a_{0}$ is the inclination. We call $\alpha_{0}$ the reduced inclination of the ray at incidence. At emergence the ray has state $\rho$ defined similarly. The transference $\mathbf{S}$ of $\mathrm{S}$ is an operator that changes $\boldsymbol{\rho}_{0}$ across $\mathrm{S}$ to $\boldsymbol{\rho}$ according to ${ }^{30,37}$

$\mathbf{S} \boldsymbol{\rho}_{0}=\boldsymbol{\rho}$,

which is equivalent to the pair of scalar equations

$$
\begin{aligned}
& A y_{0}+B \alpha_{0}=y \\
& C y_{0}+D \alpha_{0}=\alpha .
\end{aligned}
$$

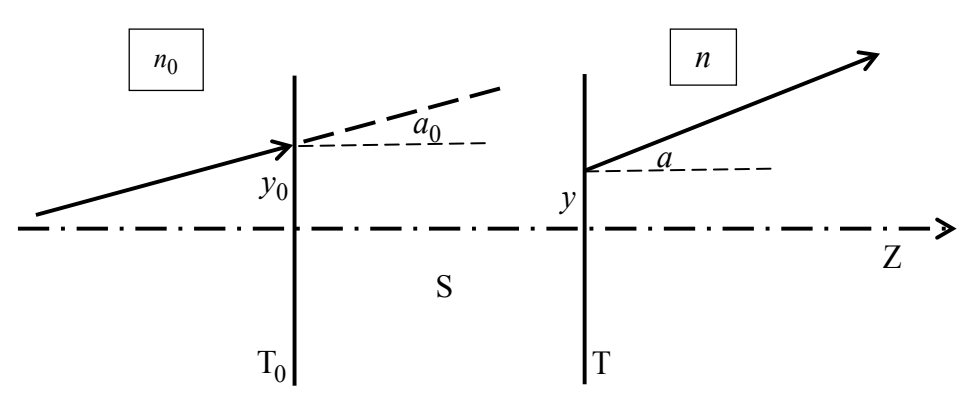

Figure 1 An arbitrary Gaussian optical system S lies between entrance plane $T_{0}$ and exit plane $T$. None of its refracting elements is shown. All elements are centred on the optical axis $Z$. A ray enters $S$ with transverse position $y_{0}$ and inclination $a_{0}$ and emerges with transverse position $y$ and inclination $a$. The ray is confined to the plane of the paper; $Z$ lies in the same plane. The media before and after the system have indices of refraction $n_{0}$ and $n$.

We now generalize these equations for linear optics; surfaces need not be invariant under rotation about the optical axis, that is, they may be astigmatic. Transverse position $y_{0}$ becomes a vector ${ }^{30,37}$

$\mathbf{y}_{0}=\left(\begin{array}{l}y_{10} \\ y_{20}\end{array}\right)$

with Cartesian coordinates $y_{10}$ and $y_{20}$ which we usually take as horizontal and vertical components respectively, and similarly for $\mathbf{y}, \mathbf{a}_{0}, \mathbf{a}, \boldsymbol{\alpha}_{0}$ and $\boldsymbol{\alpha}$. $\mathbf{y}_{0}$ and $\mathbf{y}$ are the transverse positions, $\mathbf{a}_{0}$ and $\mathbf{a}$ the inclinations and $\boldsymbol{\alpha}_{0}$ and $\boldsymbol{\alpha}$ the reduced inclinations of the ray at incidence and emergence. The components of $\mathbf{a}_{0}$ and $\mathbf{a}$ are also called direction cosines ${ }^{37}$ and the components of $\boldsymbol{\alpha}_{0}$ and $\boldsymbol{\alpha}$ are also known as optical direction cosines ${ }^{37}$. The incident state of the ray (Equation 10) generalizes to the $4 \times 1$ partitioned matrix

$\boldsymbol{\rho}_{0}=\left(\begin{array}{l}\mathbf{y}_{0} \\ \boldsymbol{\alpha}_{0}\end{array}\right)$

and similarly for the emergent state $\boldsymbol{\rho}$. Equation 12 retains the same form. Scalar Equations 13 and 14 become the matrix equations

$\mathbf{A} \mathbf{y}_{0}+\mathbf{B} \boldsymbol{\alpha}_{0}=\mathbf{y}$

$\mathbf{C} \mathbf{y}_{0}+\mathbf{D} \boldsymbol{\alpha}_{0}=\boldsymbol{\alpha}$.

Linear optics includes Gaussian optics as a special case. In the absence of astigmatism the fundamental properties become scalar matrices; we can write 
$\mathbf{A}=A \mathbf{I}$

for example.

\section{Systems in series}

Consider two optical systems $\mathrm{S}_{1}$ and $\mathrm{S}_{2}$ with transferences $\mathbf{S}_{1}$ and $\mathbf{S}_{2}$ respectively. Suppose they are juxtaposed to form a compound system. Light traverses $S_{1}$ first and then $S_{2}$. It follows from Equation 12 that the compound system has transference ${ }^{30,37}$

$\mathbf{S}=\mathbf{S}_{2} \mathbf{S}_{1}$.

In general the transference of a compound system is the product of the transferences of component systems in reverse order.

Two elementary systems are a homogenous gap and a single refracting surface. In Gaussian optics their transferences are a $^{30,37}$

$\mathbf{S}=\left(\begin{array}{ll}1 & \zeta \\ 0 & 1\end{array}\right)$

and

$\mathbf{S}=\left(\begin{array}{ll}1 & 0 \\ C & 1\end{array}\right)$

respectively. $\zeta=z / n$ is the reduced width of the gap, $z$ the actual width and $n$ the index of refraction. $C=-F$ is the divergence and $F$ the dioptric power of the surface.

\section{Object and image}

Suppose an object point $\mathrm{O}$ maps to an image point I through a Gaussian system S (Figure 2). Relative to entrance plane $T_{0}$ the longitudinal position of $\mathrm{O}$ is $z_{\mathrm{O}}$. Consider the compound system $\mathrm{S}_{\mathrm{D}}$ from the object plane $T_{O}$ to the image plane $T_{I}$. It consists of a homogeneous gap of width $-z_{\mathrm{O}}$ (the minus sign because $z_{\mathrm{O}}$ is negative), system $\mathrm{S}$ (with transference S) and a homogeneous gap of width $z_{\mathrm{I}}$. It follows from Equation 20 that the compound system has transference

$\mathbf{S}_{\mathrm{D}}=\left(\begin{array}{cc}1 & \zeta_{\mathrm{I}} \\ 0 & 1\end{array}\right)\left(\begin{array}{ll}A & B \\ C & D\end{array}\right)\left(\begin{array}{cc}1 & -\zeta_{\mathrm{O}} \\ 0 & 1\end{array}\right)$

where $\zeta_{\mathrm{O}}=z_{\mathrm{O}} / n_{0}$ and $\zeta_{\mathrm{I}}=z_{\mathrm{I}} / n$. Multiplying out we obtain

$\mathbf{S}_{\mathrm{D}}=\left(\begin{array}{cc}A+\zeta_{\mathrm{I}} C & B+\zeta_{\mathrm{I}} D-\left(A+\zeta_{\mathrm{I}} C\right) \zeta_{\mathrm{O}} \\ C & D-C \zeta_{\mathrm{O}}\end{array}\right)$.

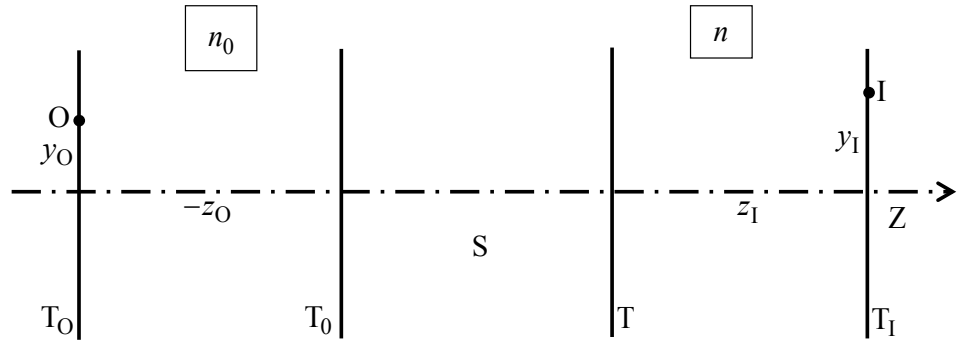

Figure 2 An object point $\mathrm{O}$ forms an image point I through a Gaussian system $\mathrm{S}$.

We now apply Equation 13 but across compound system $\mathrm{S}_{\mathrm{D}}$. Because every ray from $\mathrm{O}$ arrives at the same point I it follows that the disjugacy $B_{\mathrm{D}}$ of $\mathrm{S}_{\mathrm{D}}$ must be zero. $\mathrm{S}_{\mathrm{D}}$ is a conjugate system. Hence, from Equation 24,

$B+\zeta_{\mathrm{I}} D-\left(A+\zeta_{\mathrm{I}} C\right) \zeta_{\mathrm{O}}=0$

from which it follows that

$\zeta_{\mathrm{I}}=\frac{A \zeta_{\mathrm{O}}-B}{-C \zeta_{\mathrm{O}}+D}$

\section{Astigmatism}

In linear optics the transferences of the homogeneous gap and refracting surface are ${ }^{30,37}$

$\mathbf{S}=\left(\begin{array}{cc}\mathbf{I} & \boldsymbol{\zeta} \\ \mathbf{O} & \mathbf{I}\end{array}\right)$

and

$\mathbf{S}=\left(\begin{array}{ll}\mathbf{I} & \mathbf{O} \\ \mathbf{C} & \mathbf{I}\end{array}\right)$

respectively. Here $\mathbf{C}=-\mathbf{F}$ where $\mathbf{F}$ is the (symmetric) dioptric power matrix of the possibly-astigmatic surface. Using the notation introduced elsewhere ${ }^{54}$ we can write the power of the surface in principal meridional form as $F_{1}\{\theta\} F_{2}\left\{\theta+90^{\circ}\right\}$. (We read this as ' $F_{1}$ along $\theta$ and $F_{2}$ along $\theta+90^{\circ}$ '.) We sometimes abbreviate this to $F_{1}\{\theta\} F_{2}$. The dioptric power matrix is given by

$\mathbf{F}=\left(\begin{array}{ll}f_{11} & f_{12} \\ f_{11} & f_{12}\end{array}\right)$

where $^{25}$

$f_{11}=F_{1} \cos ^{2} \theta+F_{2} \sin ^{2} \theta$

$f_{12}=f_{21}=\left(F_{1}-F_{2}\right) \sin \theta \cos \theta$ 
$f_{21}=F_{1} \sin ^{2} \theta+F_{2} \cos ^{2} \theta$.

Equivalent equations are given by others ${ }^{20}$. As for Gaussian optics the transferences of systems compounded of gaps and surfaces can be obtained by multiplying elementary transferences (Equations 27 and 28) in reverse order.

\section{Thick spectacle lens}

A thick possibly-bitoric spectacle lens in front of the eye constitutes an instrument $S_{C}$ of four elements: in order they are a refracting surface of power $\mathbf{F}_{1}$, a homogeneous gap (the body of the lens) of reduced width $\zeta_{2}$, a second refracting surface of power $\mathbf{F}_{3}$ and a second homogeneous gap (between spectacle lens and eye) of reduced width $\zeta_{4}$. The transference is

$\mathbf{S}_{\mathrm{C}}=\left(\begin{array}{cc}\mathbf{I} & \zeta_{4} \mathbf{I} \\ \mathbf{O} & \mathbf{I}\end{array}\right)\left(\begin{array}{cc}\mathbf{I} & \mathbf{0} \\ \mathbf{C}_{3} & \mathbf{I}\end{array}\right)\left(\begin{array}{cc}\mathbf{I} & \zeta_{2} \mathbf{I} \\ \mathbf{O} & \mathbf{I}\end{array}\right)\left(\begin{array}{cc}\mathbf{I} & \mathbf{O} \\ \mathbf{C}_{1} & \mathbf{I}\end{array}\right)$

that is,

$$
\begin{aligned}
& \mathbf{S}_{\mathrm{C}}= \\
& \left(\begin{array}{cc}
\mathbf{I}+\zeta_{2} \mathbf{C}_{1}+\zeta_{4}\left(\mathbf{C}_{3}\left(\mathbf{I}+\zeta_{2} \mathbf{C}_{1}\right)+\mathbf{C}_{1}\right) & \zeta_{2} \mathbf{I}+\zeta_{4}\left(\mathbf{C}_{3} \zeta_{2}+\mathbf{I}\right) \\
\mathbf{C}_{3}\left(\mathbf{I}+\zeta_{2} \mathbf{C}_{1}\right)+\mathbf{C}_{1} & \mathbf{C}_{3} \zeta_{2}+\mathbf{I}
\end{array}\right) .
\end{aligned}
$$

\section{Condition for sharp retinal images}

Consider an emmetropic eye in linear optics. Its transference $\mathbf{S}$ is given by Equation 3. Rays from a distant object point $\mathrm{O}$ enter the eye with different transverse positions $\mathbf{y}_{0}$ and yet arrive at the same point (transverse position $\mathbf{y}$ ) on the retina it follows from Equation 17 that

$$
\mathbf{A}=\mathbf{O} \text {. }
$$

Suppose a spectacle lens or other device compensates for the refractive error of an ametropic eye. Then the compound system of device and eye satisfies Equation 35. Equation 35 is the necessary and sufficient condition for sharp retinal images. Furthermore Equation 9 results in

$$
\mathbf{B}=-\mathbf{C}^{-\mathrm{T}}
$$

and, hence, Equation 17 becomes

$$
-\mathbf{C}^{-\mathrm{T}} \boldsymbol{\alpha}_{0}=\mathbf{y} \text {. }
$$

A system for which Equation 35 holds we call exitplane focal.

\section{Condition for sharp undistorted images}

Equation 37 is a linear mapping from $\boldsymbol{\alpha}_{0}$ to $\mathbf{y}$. It shows that, although the retinal image is sharp, it may be distorted. In general a distant circle maps to an ellipse on the retina. It maps to a circle if and only if $\mathbf{C}^{-\mathrm{T}}$ is a scalar multiple of $\mathbf{R}$ where $\mathbf{R}$ is either a rotation matrix

$\mathbf{R}_{\theta}=\left(\begin{array}{cc}\cos \theta & -\sin \theta \\ \sin \theta & \cos \theta\end{array}\right)$

or a reflection matrix

$\overline{\mathbf{R}}_{\theta}=\left(\begin{array}{cc}\sin 2 \theta & \cos 2 \theta \\ \cos 2 \theta & -\sin 2 \theta\end{array}\right)$.

Because $\mathbf{R}^{-T}$ is itself a rotation or reflection matrix we can say that the object circle maps to an image circle on the retina if and only if $\mathbf{C}$ or $\mathbf{F}$ is a scalar multiple of $\mathbf{R}$, that is

$\mathbf{C}=m \mathbf{R}$

for a scalar $m$. Thus, in addition to Equation 35, there are two conditions for sharp undistorted images on the retina: either (a) the diagonal elements of $\mathbf{C}$ are equal and the off-diagonal elements are equal in magnitude but opposite in sign (based on Equation 38) or (b) the off-diagonal elements are equal and the diagonal elements are equal in magnitude but opposite in sign (Equation 39).

Suppose there is an observer beyond the plane of the distant circle who traces the object circle in a clockwise sense. The image circle on the retina would be traced in the same sense if condition (a) were satisfied and in the opposite sense if condition (b) were satisfied. We express this by saying that condition (a) preserves chirality and condition (b) reverses chirality.

For the naked eye the divergence $\mathbf{C}$ (or power $\mathbf{F}$ ) is usually not very different from a scalar matrix. It is quite possible for an eye to satisfy condition (a) but it seems inconceivable for an eye to satisfy condition (b). For eyes, then, it seems safe to disregard condition (b) and conclude that condition (a) is the only condition in practice for sharp undistorted retinal images. One expects the same to be true of the eye compensated by 
a contact or spectacle lens. (In Footnote [82] a thick lens is described which satisfies condition (b) but is totally unrealistic.) However it is quite possible for condition (b) to be satisfied by the compound system of eye and more complicated optical instrument.

Condition for sharp, undistorted and unrotated images

An eye satisfying Equation 35 and condition (a) is emmetropic and retinal images of distant objects are undistorted. However they are rotated through angle $\theta$. For the image to be sharp, undistorted and unrotated it must be that $\theta=0$ in which case $\mathbf{R}_{\theta}=\mathbf{I}$; in other words $\mathbf{C}$ and $\mathbf{F}$ must be scalar matrices. Usually they are close to scalar matrices and, so, rotations are likely to be small.

\section{Inverse transference}

Because of its symplecticity the transference is . always invertible, the inverse being ${ }^{43,} 47$

$\mathbf{S}^{-1}=\left(\begin{array}{cc}\mathbf{D}^{\mathrm{T}} & -\mathbf{B}^{\mathrm{T}} \\ -\mathbf{C}^{\mathrm{T}} & \mathbf{A}^{\mathrm{T}}\end{array}\right)$

From Equation 20 we have

$\mathbf{S}^{-1}=\mathbf{S}_{1}^{-1} \mathbf{S}_{2}^{-1}$

which shows that for a compound system the order of multiplication of inverse transferences is the same as order of component systems.

As an operator the inverse transference gives the incident state of a ray in terms of its emergent state:

$\mathbf{S}^{-1} \boldsymbol{\rho}=\boldsymbol{\rho}_{0}$

Condition for compensation of refractive error by means of a thin lens

Consider the compound system of eye and thin lens in front of it. If the eye has transference given by Equation 3 and the lens has divergence $\mathbf{C}_{1}$ (a symmetric matrix) and is located at reduced distance $\zeta_{1}$ in front of the eye then the compound system has transference

$$
\left(\begin{array}{ll}
\mathbf{A} & \mathbf{B} \\
\mathbf{C} & \mathbf{D}
\end{array}\right)\left(\begin{array}{cc}
\mathbf{I} & \mathbf{I} \zeta_{1} \\
\mathbf{O} & \mathbf{I}
\end{array}\right)\left(\begin{array}{cc}
\mathbf{I} & \mathbf{O} \\
\mathbf{C}_{1} & \mathbf{I}
\end{array}\right)
$$

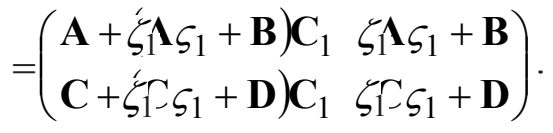

The compound system is exit-plane focal if Equation 35 is satisfied, that is,

$$
\mathbf{A}+\left(\mathbf{A} \zeta_{1}+\mathbf{B}\right) \mathbf{C}_{1}=\mathbf{O}
$$

or

$$
\mathbf{C}_{1}=-\left(\mathbf{I} \zeta_{1}+\mathbf{A}^{-1} \mathbf{B}\right)^{-1}
$$

Because of symplecticity, $\mathbf{A}^{-1} \mathbf{B}$ is symmetric ${ }^{47}$ and, hence, $\mathbf{C}_{1}$ is symmetric. Choosing the vertex distance fixes $\zeta_{1}$ and hence the divergence $\mathbf{C}_{1}$ of the compensating thin lens.

For the special case in which $\zeta_{1}=0$ Equation 46 gives $\mathbf{C}_{1}=-\mathbf{B}^{-1} \mathbf{A}$. Hence the corneal-plane refractive compensation is ${ }^{40}$

$\mathbf{F}_{0}=\mathbf{B}^{-1} \mathbf{A}$.

\section{Condition for undistorted compensation}

Suppose an optical device with transference $\mathbf{S}_{\mathrm{C}}$ is placed in front of the eye. The compound system has transference

$$
\left(\begin{array}{ll}
\mathbf{A} & \mathbf{B} \\
\mathbf{C} & \mathbf{D}
\end{array}\right)\left(\begin{array}{ll}
\mathbf{A}_{\mathrm{C}} & \mathbf{B}_{\mathrm{C}} \\
\mathbf{C}_{\mathrm{C}} & \mathbf{D}_{\mathrm{C}}
\end{array}\right)=\left(\begin{array}{ll}
\mathbf{A} \mathbf{A}_{\mathrm{C}}+\mathbf{B} \mathbf{C}_{\mathrm{C}} & \mathbf{A B} \mathbf{B}_{\mathrm{C}}+\mathbf{B} \mathbf{D}_{\mathrm{C}} \\
\mathbf{C} \mathbf{A}_{\mathrm{C}}+\mathbf{D} \mathbf{C}_{\mathrm{C}} & \mathbf{C B} \mathbf{B}_{\mathrm{C}}+\mathbf{D} \mathbf{D}_{\mathrm{C}}
\end{array}\right) .
$$

This system must satisfy both Equation 35 and Equation 40, that is,

$$
\mathbf{A} \mathbf{A}_{\mathrm{C}}+\mathbf{B} \mathbf{C}_{\mathrm{C}}=\mathbf{O}
$$

and

$\mathbf{C} \mathbf{A}_{\mathrm{C}}+\mathbf{D} \mathbf{C}_{\mathrm{C}}=m \mathbf{R}$.

Making use of Equation 47 we can write Equation 49 as

$$
\mathbf{F}_{0} \mathbf{A}_{\mathrm{C}}+\mathbf{C}_{\mathrm{C}}=\mathbf{O}
$$

From Equation 49 we obtain

$\mathbf{C}_{\mathrm{C}}=-\mathbf{B}^{-1} \mathbf{A} \mathbf{A}_{\mathrm{C}}$.

Hence Equation 50 becomes

$\left(\mathbf{C}-\mathbf{D B}{ }^{-1} \mathbf{A}\right) \mathbf{A}_{\mathrm{C}}=m \mathbf{R}$.

We recognize the coefficient of $\mathbf{A}_{\mathrm{C}}$ as the Schur 
complement ${ }^{47,55,56}$ of $\mathbf{C}$ in the symplectic matrix $\mathbf{S}$. Hence $^{47,57}$ Equation 53 simplifies to

$-\mathbf{B}^{-\mathrm{T}} \mathbf{A}_{\mathrm{C}}=m \mathbf{R}$

and so

$$
\mathbf{A}_{\mathrm{C}}=-m \mathbf{B}^{\mathrm{T}} \mathbf{R}
$$

We substitute into Equation 49 and use the fact that $\mathbf{A B}^{\mathrm{T}}$ is symmetric to obtain

$$
\mathbf{C}_{\mathrm{C}}=m \mathbf{A}^{\mathrm{T}} \mathbf{R} .
$$

\section{Le Grand's appendix}

The brief summary above of the matrix approach to Gaussian and linear optics provides a coherent framework against which we can now examine Le Grand's appendix. A translation is given in the appendix of this paper. It is a translation of the appendix in the first edition of Le Grand's book ${ }^{26}$. A few minor changes made in the third edition ${ }^{27}$ are noted. Superscript numbers, as in $\bullet^{n}$, in the translation continue to refer to references listed under References at the end of the paper; superscript numbers in square brackets, as in $\bullet^{[n]}$, refer to detailed annotations listed as footnotes in the translation. Le Grand's original equation numbering has been preserved including the fact that his equation numbers appear to the left of equations; the numbers were changed in the third edition.

We turn now to the more important questions concerning Le Grand's appendix. Many minor matters, including a small number obvious typographical errors, will be left to the footnotes.

\section{Le Grand on the transference}

The first reference Le Grand makes to what appears to be a transference is his unnumbered equation after superscript [26]. Comparison of the matrix on right-hand side with Equation 1 suggests that the matrix is a transference in Gaussian optics. This is reinforced by Le Grand's Equation (108) in the light of the symplectic requirement, Equation 2. More particularly, comparison of the left-hand matrix with Equation 22 suggests that it is the transference of a refracting surface. However the signs of the bottom-left entries differ.

At first sight the second matrix at superscript [30] appears to be the transference of a homogeneous gap of reduced thickness $-\delta$ (compare Equation 21) and the equation appears to represent the transference of a compound system consisting of the homogeneous gap followed by a system $S_{1}$ obtained by multiplication in reverse order according to Equation 20. Similar remarks apply to the equations at [32] and [34].

In actual fact the negative sign gives Le Grand's matrix away, not as the transference $\mathbf{S}$ of a gap of reduced thickness $-\delta$ but the inverse transference $\mathbf{S}^{-1}$ of a gap of thickness $\delta$. Similarly Le Grand's matrix for the refracting surface is actually the inverse transference of the surface.

While transferences multiply in reverse order of component elements (Equation 20) inverse transferences multiply in the same order as the component elements (Equation 42). This accounts for the order of multiplication in Equation (110) at superscript [34], in the numerical example that follows it and elsewhere in the appendix.

In the presence of astigmatism Le Grand writes the $4 \times 4$ matrix $\mathbf{M}$ (at [63]) with the six equations among the entries (at [67]). That it is also an inverse transference rather than a transference is clear from the matrix for a gap (at [70]) and the order of multiplication at [71]. It is also clear at [69] where the bottom-left block of the matrix for an astigmatic refracting surface consists of powers and not their negatives. The inverse of a symplectic matrix is also symplectic $^{43}$ which is why there should be the six equations Le Grand refers to as mentioned above (just after Equation 4) for a transference.

The inverse transference is an operator that operates on the emergent state of a ray to give the incident state for that ray for a given optical system (Equation 43). However nowhere in his appendix does Le Grand hint at such an interpretation. One gets the impression that he sees the matrix merely as a convenient mathematical device for determining optical properties of compound systems. The matrix is that of course, but its nature as an operator which represents the way the system operates on light seems to be an important feature of the inverse transference, as of the transference itself, which does not come across here.

Instead of thinking of the transference as the property of a particular system that operates on a ray 
traversing it Le Grand's thinking is in terms of object and image. This is clear from his frequent reference to origins (see just before [25] and [27] for example). Origins relative to which objects and images are located need to be defined. There is less emphasis on the need to define optical systems. An optical system followed by a homogeneous gap is treated between [27] and [30] but it is not treated as a new compound system; it is treated as the same system but with the origin for images shifted downstream by the width of the gap. Of course the two approaches are equivalent but Le Grand's seems less clear.

An advantage of Le Grand's approach is the simpler equations one can obtain if one chooses the origins to be at the principal planes. He takes advantage of this fact at [17] and in the solution to Exercise 43. However this is true only for Gaussian optics; because principal planes are not usually well defined in the presence of astigmatism ${ }^{58}$ the approach does not generalize to linear optics.

\section{Le Grand on the dioptric power matrix}

The bottom-left block of an inverse transference is $-\mathbf{C}^{\mathrm{T}}$ (Equation 41 ) or, in other words, $\mathbf{F}^{\mathrm{T}}$ (Equation 4). Because of symmetry (Equation 29 and 31) the bottom-left block is simply $\mathbf{F}$ in the case of an astigmatic refracting surface. Thus the bottom-left block at [69] is the dioptric power matrix of the surface. Expressions for the entries in the block are given at [53]. While the expressions for $C$ and $B$ match those for the diagonal entries of $\mathbf{F}$ (Equations 30 and 32) Le Grand's expression for $A$ is double that of the off-diagonal entries (Equation 31). Thus if the factor $\frac{1}{2}$ is inserted then Le Grand's equations give the entries of the dioptric power matrix. Le Grand's omission of the factor must surely be a typographical slip. (In the numerical example dealt with near the end of Le Grand's appendix $A=0$, so the omission has no effect on the numerical results in this particular case.)

Just as for the transference Le Grand does not seem to think of the dioptric power matrix as an integral whole. He does not use a symbol for it. He talks instead of the three powers of a surface (at [53]) which become four powers of a system in general (at [64]). The powers are separate entities. There is no suggestion of a unified function.

\section{Le Grand on other matters}

At [72] Le Grand presents the condition that an eye or compensated eye will form sharp retinal images of distant objects. The condition consists of four equations. An explanation is not given and is certainly not obvious. Le Grand's conditions are derived in Footnote [72]; they are a direct consequence of the condition (Equation 35) given above.

In the presence of astigmatism the sharp retinal image is usually distorted. At [75] Le Grand presents a supplementary condition, also without explanation, which needs to be satisfied if the image is undistorted. His condition takes the form of a single scalar equation. The required conditions are derived in Footnote [75]; they take the form of three scalar equations in addition to Le Grand's single equation. The basis of the derivation being Equation 40 and the two associated conditions (a) and (b). One obtains a pair of equations (Equations 49 and 50) which can be reduced to Equations 55 and 56. There is much more to the problem than presented by Le Grand; strictly speaking his condition is neither necessary nor sufficient. It is possible to have systems which produce sharp undistorted images of distant objects but which do not satisfy Le Grand's condition. However it seems unlikely for such cases to be encountered in conventional applications in optometry. If these cases are neglected Le Grand's condition becomes necessary. Strictly speaking it is not a sufficient condition although one expects the additional requirement to be satisfied automatically by most eyes.

It is perhaps worth noting that the numerical example (at [83] to [85]) which Le Grand selects to illustrate the use of his equations at [82] can be solved simply by means of Gaussian optics applied once along each principal meridional plane. That is because there are no obliquely-crossing principal meridians. However his equations are actually more powerful than he illustrates; they can handle obliquely-crossing meridians without any difficulty which is not possible with Gaussian optics.

\section{Concluding remarks}

Authors in optometry have not always been as careful as they might have been in attributing credit. 
The recognition that the present author is no exception has motivated this study. The attempt has been to make a scholarly analysis of exactly what Le Grand had to say in his appendix about matrices in optics and, more particularly, about the dioptric power matrix and the ray transference. In order to obviate any possible ambiguity I have thought it appropriate to provide detailed and exhaustive annotations to the translation.

In view of what we have seen here, if asked whether Le Grand described the dioptric power matrix and the transference one would have to say 'No, not in the full sense in which those concepts are now understood'. That, however, would be a misrepresentation for he certainly came very close. The missing $\frac{1}{2}$ in his expression for the off-diagonal elements of the dioptric power matric of an astigmatic refracting surface is almost certainly a typographical error and is not the issue. He seems to have thought of three separate powers rather than an integrated whole dioptric power. Le Grand worked not with the transference but its inverse; nowhere in his appendix does he deal with the inverse of his matrix (which would have been the transference) and, importantly, he does not seem to have thought of his matrix as a whole entity in its own right and more particularly as an entity that operates on the state of a ray traversing the system.

When reading Le Grand it is important to note that he takes the optical system of the eye to be from just anterior to the cornea to just posterior to the lens. The vitreous is excluded. (See Footnote [36].) The eye as optical system to which we refer in this paper includes the vitreous.

The conditions for sharp retinal images of distant objects are derived in Footnote [72]; they are identical to the conditions that Le Grand presents without justification. The conditions for sharp images that are also undistorted are derived in Footnote [75]; here Le Grand's condition does not tell the whole story. Nevertheless it seems safe to say his condition tells most of the story at least in the context of conventional optometric applications. A fuller analysis of sharp and undistorted retinal images, including for near objects, has recently appeared elsewhere ${ }^{59}$.

In looking carefully at Le Grand's thinking, as we have tried to do here, we become more conscious still of those scholars before him who too have not always been given their due. There are Herzberger's important contributions ${ }^{60-62}$ of the 1930s, Smith's ${ }^{63-68}$ of the 1920s and many others all the way back to Gauss $^{69}$ and, perhaps, before. For, reading these older works, one is frequently surprised, by ideas one thought were new. "The wind goeth toward the south, and turneth about unto the north; it whirleth about continually, and the wind returneth again according to his circuits. / ... / The thing that hath been, it is that which shall be; and that which is done is that which shall be done: and there is no new thing under the sun. / Is there anything whereof it may be said, See, this is new? It hath been already of old time, which was before us." (Ecclesiates 1 9-10).

\section{Acknowledgements}

I gratefully acknowledge the assistance of the following: Akshita Dhurmah, who advised on the translation, and Tanya Evans and Radboud DHM van Gool, for discussions on the ophthalmic optics, all three of the Department of Optometry, University of Johannesburg; Gerald Westheimer, Division of Neurobiology, University of California, Berkeley, CA, who put me in touch with Kenneth Knoblauch, Inserm, Stem-Cell and Brain Research Institute, Bron, France, who put me in touch with Françoise Viénot, Centre for Research on Conservation of Collections, National Museum of Natural History, Paris; Pierre Lemonde, Attaché for Science and Technology, and Lucky Kunene, Cooperation and Cultural Action Service, Embassy of France in South Africa; Thorsten Schneider, editor at Springer; Larry N Thibos, School of Optometry, Indiana University, Bloomington, IN; Keith B. MacAdam, Department of Physics and Astronomy, University of Kentucky, Lexington, KY; and the National Research Foundation of South Africa for financial support.

I thank an anonymous reviewer for an insightful analysis and for drawing my attention to the existence of a Spanish translation of Le Grand's appendix. Acquisition of a copy, and the assistance of a Spanish speaker familiar with ophthalmic optics, may give me more insight into Le Grand's work. 


\section{Appendix \\ 'The arithmetic of matrices in optics ${ }^{\text {'[1] }}$ by Yves Le Grand $^{[2]}$}

In preparing this volume I have strived to keep the mathematical development at a level as elementary as possible, and in terms of optical formulae most widely used in classical education. However, the same problems can be solved with greater elegance and generality by employing certain algebraic symbols, matrices, the use of which is current in contemporary physics. We will consider here only square matrices.

One calls a matrix of order $n$ a table formed of $n$ rows and $n$ columns, consisting thus of $n^{2}$ quantities arranged as follows: ${ }^{[3]}$

$$
\left(\begin{array}{ccccc}
a_{11} & a_{12} & a_{13} & \cdots & a_{1 n} \\
a_{21} & a_{22} & a_{23} & \cdots & a_{2 n} \\
\vdots & \vdots & \vdots & \vdots & \vdots \\
a_{n 1} & a_{n 2} & a_{n 3} & \cdots & a_{n n}
\end{array}\right)
$$

In the term $a_{p g}$ of the matrix index $p$ designates the number of the row and index $q$ the number of the column. For what follows the only property of matrices which we will need concerns multiplication: one defines the product of two matrices of order $n$ as a third matrix also of order $n$ $\left(\begin{array}{ccccc}a_{11} & a_{12} & a_{13} & \cdots & a_{1 n} \\ a_{21} & a_{22} & a_{23} & \cdots & a_{2 n} \\ \vdots & \vdots & \vdots & \vdots & \vdots \\ a_{n 1} & a_{n 2} & a_{n 3} & \cdots & a_{n n}\end{array}\right)\left(\begin{array}{ccccc}b_{11} & b_{12} & b_{13} & \cdots & b_{1 n} \\ b_{21} & b_{22} & b_{23} & \cdots & b_{2 n} \\ \vdots & \vdots & \vdots & \vdots & \vdots \\ b_{n 1} & b_{n 2} & b_{n 3} & \cdots & b_{n n}\end{array}\right)$

$$
=\left(\begin{array}{ccccc}
c_{11} & c_{12} & c_{13} & \cdots & c_{1 n} \\
c_{21} & c_{22} & c_{23} & \cdots & c_{2 n} \\
\vdots & \vdots & \vdots & \vdots & \vdots \\
c_{n 1} & c_{n 2} & c_{n 3} & \cdots & c_{n n}
\end{array}\right)
$$

the terms of the matrix product obtained by the $n^{2}$ relations ${ }^{[4]}$

$$
c_{p q}=\sum_{k=1}^{k=n} a_{p k} b_{k q} \text {; }
$$

that is to say that any term $c$ is obtained from the $a$ s of the same row and the $b$ s of the same column as the sum of their pairwise products.

It is easy to see that the product of matrices is not commutative; one cannot reverse the factors. For example

$$
\left(\begin{array}{ll}
1 & 2 \\
3 & 4
\end{array}\right)\left(\begin{array}{ll}
5 & 6 \\
7 & 8
\end{array}\right)=\left(\begin{array}{ll}
19 & 22 \\
43 & 50
\end{array}\right),
$$

while

$$
\left(\begin{array}{ll}
5 & 6 \\
7 & 8
\end{array}\right)\left(\begin{array}{ll}
1 & 2 \\
3 & 4
\end{array}\right)=\left(\begin{array}{ll}
23 & 34 \\
31 & 46
\end{array}\right) .
$$

However this operation is associative: in the product of several matrices one can group them at will provided one does not change the order:

$$
\left(\mathbf{M}_{1} \mathbf{M}_{2}\right) \mathbf{M}_{3}=\mathbf{M}_{1}\left(\mathbf{M}_{2} \mathbf{M}_{3}\right) \text {. }^{[5]}
$$

Gauss's Coefficients. - Consider a centred ${ }^{[6]}$ system on the axis of which we choose any two origins; the abscissas $x$ and $x^{\prime}$ of two conjugate points

[1]. This is a translation by WFH from the original French of Annexe II: Le Calcul des Matrices en Optique, pp 322-328, and parts of pp 332 \& 341 of the 1 st edn of the book ${ }^{26}$ that LG (Le Grand) originally wrote 'in 1945 as a textbook for the students of the Institute of Optics in Paris ${ }^{28}$. The 3rd edn of the book ${ }^{27}$ appeared in English translation ${ }^{29}$ in 1980. Although Annexe II appears with minor changes on pp 364-381 of the 3rd edn, it is omitted in the English version.

[2]. The title page of the $1 \mathrm{st}^{\mathrm{edn}}{ }^{26}$ is headed, in translation, 'Yves LE GRAND/ Deputy Director of the Physical Laboratory of the National Museum of Natural History/ Head of Conferences at the Polytechnic School'. In the 3rd edn ${ }^{27}$ LG is described as 'Professor of the National Museum of Natural History/ Examiner of students of the Polytechnic School'. From the French version of Wikipedia (27 March 2013$)$ we learn that LG (1908-1986) obtained his doctorate in 1936 with a thesis on dispersion of light in the eye. The same year he joined the Laboratory of Physics Applied to Living Beings of the National Museum of Natural History of which he later became Director. From 1942 he taught physiological optics at the School of Optics. In 1966 he was elected president of the French Society of Physiological Optics which he had helped to create.

[3]. Throughout LG uses straight lines for matrices (as now often used for determinants) instead of the curved brackets used here. There is a typographical error in the $3 \operatorname{rd~edn}^{27}$ : the 2 nd and 3rd entries $a_{n 2}$ and $a_{n 3}$ of the bottom row of this matrix are each given as $a_{n 1}$.

[4]. The last subscript $q$ in this equation is incorrectly written $p$ in the $3 \mathrm{rd} \mathrm{edn}^{27}$.

[5]. LG uses multiplication signs $(\times)$ and $\mathbf{M}$ is represented by $\mathbf{M}$.

[6]. By a centred system LG means what is often called a symmetric system. Refracting elements of the system are invariant under rotation about a common axis, the optical axis. 
projected onto the axis ${ }^{[7]}$ are linked by a homographic transformation ${ }^{[8]}$ which we will write in the form: ${ }^{[9]}$

(107) $x^{\prime}=\frac{c x+d}{a x+b}$.

The quantities ${ }^{[10]} a, b, c, d$ are evidently determined only to a constant factor since the relation still holds when one multiplies them by the same number. One will therefore be able to impose an arbitrary relation among these quantities.

The longitudinal magnification is expressed by:

$$
\frac{\mathrm{d} x^{\prime}}{\mathrm{d} x}=\frac{b-d}{(a x+b)^{2}}
$$

If the extreme media have an index of refraction of 1 the transverse magnification $y^{\prime} / y$ is such that ${ }^{[11]}$ $\left(\frac{y^{\prime}}{y}\right)^{2}=\frac{\mathrm{d} x^{\prime}}{\mathrm{d} x}$

consequently, if we choose the condition $(108)^{[12]} \quad b c-a d=1$

as arbitrary relation, then it becomes (one proves that it necessarily takes the sign +$)^{[13]}$

$(109)^{[14]}$

$$
\frac{y^{\prime}}{y}=\frac{1}{a x+b} .
$$

Quantities $a, b, c, d$, are called Gauss's coefficients; $a$ is a power, $b$ and $c$ numbers, and $d$ a length ${ }^{[15]}$. From a knowledge of these coefficients, one immediately deduces the cardinal elements of the system $^{[16]}$; the abscissas of the principal points are obtained by putting $y^{\prime} / y=1$ in equation (109), which gives $x_{0}=(1-b) / a$ and by substitution in (107) $x_{0}^{\prime}=(c-1) / a$. If one takes these points as origins,

[7]. The picture is as follows:

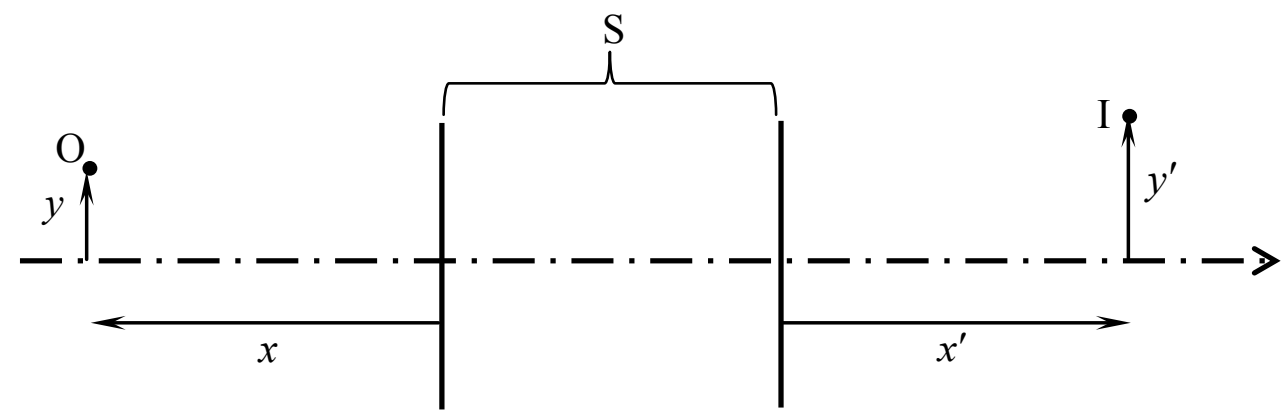

Object point $\mathrm{O}$ maps to an image point I through system $\mathrm{S}$.

[8]. Also called a Möbius transformation.

[9]. The equation number is given as (AII-1) in the 3rd edn. Apparently this equation was better known in the past than it is today. A derivation was given by Pendlebury ${ }^{70}$. We derive it above (see Eq 26).

[10]. After Eq (108) is applied these quantities become entries of the transference (Eq 1) of system S. In particular $a=-C, b=D, c=A$, $d=-B$

[11]. The 3rd edn has 'see exercise 3' in brackets. The compound system from object plane to image plane has transference $\left(\begin{array}{cc}A+C x^{\prime} & 0 \\ C & D-C x\end{array}\right)($ Eqs $24 \& 25)$. Application of Eq 13 to the compound system shows that $\left(A+C x^{\prime}\right) y=y^{\prime}$. Because of symplecticity (Eq

2) $\left(A+C x^{\prime}\right)(D-C x)=1$. Hence, the transverse magnification is $y^{\prime} / y=A+C x^{\prime}=1 /(D-C x)$ in agreement with Eq (109). [12]. The equation number is (AII-2) in the 3rd edn. Because of Eq (108) the matrix $\left(\begin{array}{ll}b & d \\ a & c\end{array}\right)$ to be encountered below has unit determinant and
is symplectic.

[13]. The plus sign follows the closing bracket in the original. This is corrected in the $3 \mathrm{rd}$ edn.

[14]. Eq (AII-3) in the 3rd edn.

[15]. Although $d$ has the dimension length it does not seem to be entirely accurate to call $d$ a length. For an air gap it is the negative of the length of the gap. In general it is the negative of the disjugacy. What LG calls Gauss's coefficients are the entries of the inverse of the transference (see Footnote 26).

[16]. Because of the assumption of unit refractive index outside the system (see 2nd sentence of this paragraph) the nodal points coincide with the principal points expressions for the locations of which are given here. Setting $x=0$ in Eq (107) locates the emergent focal point at $x^{\prime}=d / b$. The incident focal point is obtained by setting $x^{\prime}=0$ in Eq (107), the result being $x=-d / c$. 
it happens that $b=c=1, d=0$ and equation (107) becomes $^{[17]}$

$\frac{1}{x^{\prime}}=\frac{1}{x}+a$

which shows us that $a$ is nothing other than the true power ${ }^{[18]}$ of the system. If one is satisfied with the choice of conjugate origins then $d=0$, and from (108) $b c=1 .^{[19]}$ Then equation $(107)$ is written $^{[20]}$ $\frac{1}{x^{\prime}}=\frac{b^{2}}{x}+b a$

we recover the frontal ${ }^{[21]}$ notations, $b$ designating the frontal factor ${ }^{[22]} g^{\prime}$ and $b a$ the frontal power $D_{f}^{\prime} \cdot{ }^{[23]}$ If the indices of refraction of the extreme media differ from 1 the expressions above remain valid ${ }^{[24]}$ provided that $x$ and $x^{\prime}$ designate the reduced distances of the points considered from the respective origins.

Use of matrices. - The arithmetic of matrices allows the very simple determination of Gauss's coefficients of a centred system, whatever its complexity.

1 . If the system is a refracting surface of power $D$, the origins coinciding with the apex of the surface, ${ }^{[25]}$ one

has $\frac{1}{x^{\prime}}=\frac{1}{x}+D ;$ consequently $a=D, b=c=1$, $d=0$, which can be written ${ }^{[26]}$

$\left(\begin{array}{ll}1 & 0 \\ D & 1\end{array}\right)=\left(\begin{array}{ll}b & d \\ a & c\end{array}\right)$.

[17]. The system under consideration has now changed but the symbolism remains the same. Originally the system was defined by the dashed vertical lines in the following sketch; it is now defined by the incident $\mathrm{P}$ and the emergent $\mathrm{P}^{\prime}$ principal planes whose longitudinal positions $x_{0}$ and $x_{0}^{\prime}$ are now 0 . It follows from $x_{0}=(1-b) / a$ and $x_{0}^{\prime}=(c-1) / a$ that $b=c=1 . d=0$ because the new system is conjugate.

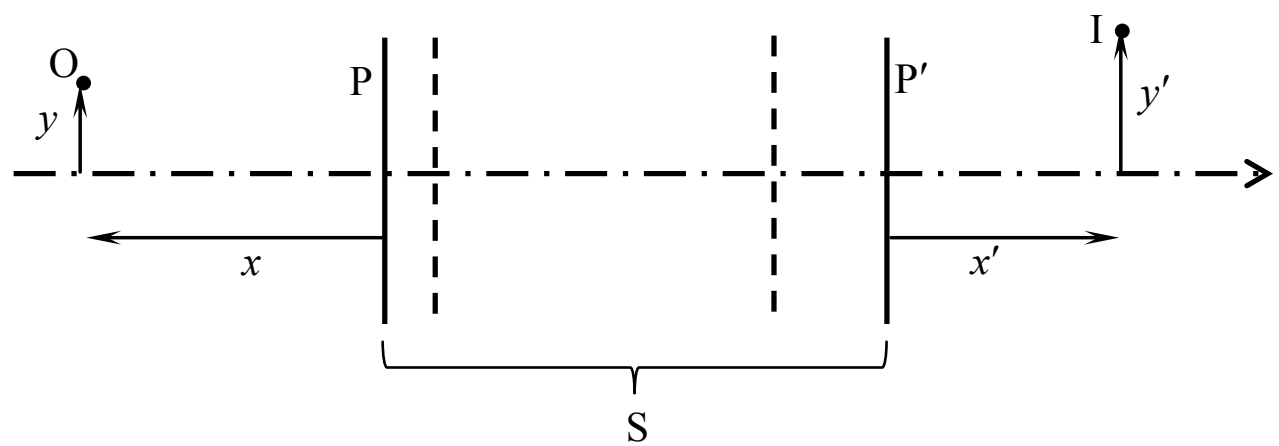

[18]. 'la puissance vraie' which is changed to 'la puissance équivalente' (equivalent power) in the 3rd edn.

[19]. Again the system changes but the symbols remain the same. $\mathrm{P}$ and $\mathrm{P}^{\prime}$ in the sketch above are not necessarily principal planes but any pair $\mathrm{Q}$ and $\mathrm{Q}^{\prime}$ of conjugate planes. $d=0$ because this new system, from $\mathrm{Q}$ to $\mathrm{Q}^{\prime}$, is also conjugate and, hence, $b c=1$ follows. LG (p 38 of Ref 26) called 1/x proximité. LG\&EH (Le Grand and El Hage) (p 13 of Ref 29) used proximity and ascribed the concept and term to Herschel in 1827. They also comment ( $\mathrm{p} 14$ ) that proximity and power are sometimes called vergence and convergence respectively, terms best avoided because of the other meanings they have in binocular vision. Presumably the reference is to John Frederick William Herschel (1792-1871, lived in South Africa 1834-1838), the son of Frederick William Herschel who discovered Uranus and who died in 1822.

[20]. The equation follows directly from Eq (107) with $d=0$ and $b c=1$. It is Eq 48 (except that $D_{f}^{\prime}$ is $D^{\prime}$ there), together with Eq 52, on p 105 of Ref 26 and Eq 1.50 on p 22, together with Eq 1.49 on p 21, of Ref 29.

[21]. LG uses frontal instead of vertex.

[22]. 'le facteur frontale' which becomes 'le facteur de forme' (form factor) in the 3rd edn.

[23]. $D_{f}^{\prime}$ is the back (or image) frontal power (p 102 of Ref 26 and p 20 of Ref 29) usually known as back-vertex power. LG (pp 102-105 of Ref 26 and pp 19-22 of Ref 29) defines 4 frontal powers: forward frontal object power (front vertex power), forward frontal image power, rear frontal object power and rear frontal image power (back vertex power) but retains only the 1 st and last. Footnote 2 on $\mathrm{p} 104$ of Ref 26 mentions that $b$ was called effectivity factor by Smith and shape factor by Ogle. In Ref 63 Smith refers to "so-called "effectivity" factors- not a very suitable name'. For Ogle see Ref 71.

[24]. 'valables' which is changed to 'exactes' (correct) in the $3 \mathrm{rd}$ edn.

[25]. We have interpreted the French un dioptre de puissance $D$ as meaning a refracting surface of power $D$. It appears that $x$ and $x$ ' here are reduced distances. If the indices of refraction either side of the surface were 1 then the power of the surface would be 0 . Dioptre meaning refracting surface is distinct from LG's dioptrie which means dioptre (diopter in American English) the unit of power. D is the symbol LG uses for power (puissance).

[26]. Using the values from Footnote [10] we obtain $\left(\begin{array}{ll}b & d \\ a & c\end{array}\right)=\left(\begin{array}{cc}D & -B \\ -C & A\end{array}\right)$. Comparing this with Eq 1 we see that LG's matrix is not the transference $\mathbf{S}$ but its inverse $\mathbf{S}^{-1}$. LG's matrix $\left(\begin{array}{ll}1 & 0 \\ D & 1\end{array}\right)$ applies to a thin system (a refracting surface or thin lens) and to any Gaussian system but taken between its principal planes. 
2. Let us displace the origin of the images by reduced from which by identification distance $\delta ;[27]$ the system ${ }^{[28]}$, which before had coefficients $a_{1}, b_{1}, c_{1}, d_{1}$, has $a, b, c, d$ such that ${ }^{[29]}$

$$
\begin{aligned}
x^{\prime} & =x_{1}^{\prime}-\delta=\frac{c_{1} x+d_{1}}{a_{1} x+b_{1}}-\delta=\frac{\left(c_{1}-a_{1} \delta\right) x+\left(d_{1}-b_{1} \delta\right)}{a_{1} x+b_{1}} \\
& =\frac{c x+d}{a x+b},
\end{aligned}
$$

from which $a=a_{1}, \quad b=b_{1}, c=c_{1}-a_{1} \delta, d=d_{1}-b_{1} \delta$ (one can verify that condition (108) is satisfied), which can be written $^{[30]}$

$\left(\begin{array}{ll}b_{1} & d_{1} \\ a_{1} & c_{1}\end{array}\right)\left(\begin{array}{cc}1 & -\delta \\ 0 & 1\end{array}\right)=\left(\begin{array}{ll}b & d \\ a & c\end{array}\right)$.

3. Let us link a second system $a_{2} b_{2} c_{2} d_{2}$ (with origins $\mathrm{O}_{2} \mathrm{O}_{2}^{\prime}$ ) to the first system $a_{1} b_{1} c_{1} d_{1}$ (with origins $\mathrm{O}_{1} \mathrm{O}_{1}^{\prime}$ ) and let us consider the resulting system abcd (with origins $\mathrm{OO}^{\prime}$ ); let us make $\mathrm{O}$ coincide with $\mathrm{O}_{1}$ and $\mathrm{O}^{\prime}$ with $\mathrm{O}_{2}^{\prime}$. We have

$x^{\prime}=\frac{c x+d}{a x+b}=\frac{c_{2} x_{1}^{\prime}+d_{2}}{a_{2} x_{1}^{\prime}+b_{2}}$, with $x_{1}^{\prime}=\frac{c_{1} x+d_{1}}{a_{1} x+b_{1}} ;{ }^{[31]}$

$a=a_{1} b_{2}+c_{1} a_{2}$

$b=b_{1} b_{2}+d_{1} a_{2}$

$c=a_{1} d_{2}+c_{1} c_{2}$

$d=b_{1} d_{2}+d_{1} c_{2}$,

which can be written ${ }^{[32]}$

$\left(\begin{array}{ll}b_{1} & d_{1} \\ a_{1} & c_{1}\end{array}\right)\left(\begin{array}{ll}b_{2} & d_{2} \\ a_{2} & c_{2}\end{array}\right)=\left(\begin{array}{ll}b & d \\ a & c\end{array}\right)$.

4. If we add to the three preceding results the associative character of matrix multiplication we obtain the following rule: consider a centred system made up of refracting surfaces of powers $D_{1}, D_{3}, D_{5}, \ldots$ separated by reduced distances $\delta_{2}, \delta_{4}, \ldots$; the resulting system, of which the object origin coincides with the apex of the first refracting surface and the image origin with the apex of the last, has Gauss's coefficients ${ }^{[33]}$ $(110)^{[34]}$

$\left(\begin{array}{cc}1 & 0 \\ D_{1} & 1\end{array}\right)\left(\begin{array}{cc}1 & -\delta_{2} \\ 0 & 1\end{array}\right)\left(\begin{array}{cc}1 & 0 \\ D_{3} & 1\end{array}\right)\left(\begin{array}{cc}1 & -\delta_{4} \\ 0 & 1\end{array}\right)\left(\begin{array}{cc}1 & 0 \\ D_{5} & 1\end{array}\right) \cdots=\left(\begin{array}{ll}b & d \\ a & c\end{array}\right)$ Applications. - Expression (110) allows the immediate recovery of the formulae associated with Gullstrand $\left.{ }^{1}\right) .{ }^{[35]}$ One can also use it for the numerical calculation

[27]. The origin for $x^{\prime}$ is shifted a longitudinal distance $n^{\prime} \delta$ as in the sketch below. $n^{\prime}$ is the index of refraction of the medium after the optical system.

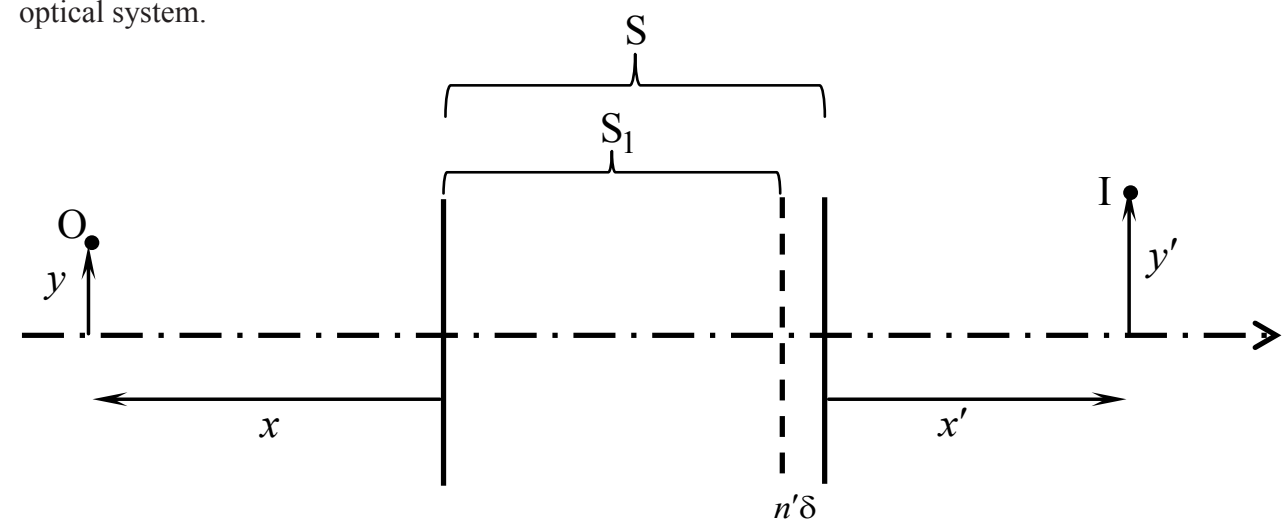

[28]. What was optical system $\mathrm{S}$ now becomes system $\mathrm{S}_{1}$. System $\mathrm{S}$ now includes system $\mathrm{S}_{1}$ and the homogeneous gap of width $n^{\prime} \delta$.

[29]. $x, x^{\prime}, x_{1}^{\prime}$ are reduced distances.

[30]. For the reader familiar with transferences the order of multiplication here appears incorrect. If the gap in the sketch has transference $\mathbf{S}_{2}$ then the transference of system $\mathrm{S}$ is $\mathbf{S}=\mathbf{S}_{2} \mathbf{S}_{1}$ (Eq 20). Because LG is multiplying inverse transferences (see Footnote [26]), his order is correct. See Eq 42.

[31]. The prime is missing from $x_{1}^{\prime}=\left(c_{1} x+d_{1}\right) /\left(a_{1} x+b_{1}\right)$ in the 3 rd edn.

[32]. See Footnote [30].

[33]. See Footnote [30].

[34]. (AII-4) in the 3rd edn.

[35]. ( ${ }^{1}$ ) refers to the footnote on the same page (p 325) which reads ' (') See exercises 43 and 44.' The exercises follow the Annexe on $\mathrm{p} 329$. 
of a complex system; for example let us apply it to the case of the theoretical non-accommodated eye: ${ }^{[36]}$

$D_{1}=48.3462 \mathrm{dt}$

$\delta_{2}=0.55 \times 10^{-3} / 1.3771=3.9939 \times 10^{-4} \mathrm{~m}$

$D_{3}=-6.1077 \mathrm{dt}$

$\delta_{4}=3.05 \times 10^{-3} / 1.3374=2.2805 \times 10^{-3} \mathrm{~m}$

$D_{5}=8.0980 \mathrm{dt}$

$\delta_{6}=4 \times 10^{-3} / 1.42=2.8169 \times 10^{-3} \mathrm{~m}$

$D_{7}=14 \mathrm{dt}$.

One calculates the successive products step by step; thus the products of the first three, the first five, and of the seven matrices equal ${ }^{[37]}$

$\left(\begin{array}{cc}1.00244 & -3.9939 \times 10^{-4} \\ 42.3564 & 0.98069\end{array}\right)$,
$\left(\begin{array}{cc}0.98069 & -2.6855 \times 10^{-3} \\ 49.5158 & 0.88410\end{array}\right)$,

$\left(\begin{array}{cc}0.90442 & -5.4480 \times 10^{-3} \\ 59.9404 & 0.74461\end{array}\right)$

respectively. The position of the object principal point will be

$\frac{1-b}{a}=\frac{1-0.90442}{59.9404}=1.5946 \times 10^{-3} \mathrm{~m} ;{ }^{[38]}$

one will find the image principal point ${ }^{[39]}$ in the same way: we obtain the values in table I (page 50) ${ }^{[40]}$.

Moreover there will be every interest in preserving the origins of Gauss's coefficients: ${ }^{[41]}$ the object origin will be the apex of the cornea, a well-defined physical ${ }^{[42]}$ point, and the image origin will be the apex of the posterior surface of the crystalline lens, which moves very little during accommodation. If the eye is corrected one will introduce the correcting refracting surfaces in front of the eye and formulae (110) and (109) will allow us very easily to calculate the dimension of the retinal image, ${ }^{[43]}$ assumed

[36]. Note that this 'eye' excludes the vitreous body. The index of refraction and width of the vitreous are not given in the Annexe. If we take the index to be 1.336 (as given in tableau 1 on p 50 of Ref 26) and the width as $24.1965 \mathrm{~mm}$ (the value calculated on p 49 of Ref 26 to make the eye emmetropic) then we find that the transference of the whole eye, including the vitreous, is $\left(\begin{array}{ccc}0.00000 & 16.6832 \times 10^{-3} \mathrm{~m} \\ -59.9404 \mathrm{D} & 0.90442\end{array}\right) \cdot$ That the top left entry is 0 shows that the eye is indeed emmetropic. The inverse of the eye's transference is $\left(\begin{array}{cc}0.90442 & -16.6832 \times 10^{-3} \mathrm{~m} \\ 59.9404 \mathrm{D} & 0.00000\end{array}\right)$. Note also that dioptres is abbreviated $d t$ and not $D$. LG uses the decimal comma rather than the decimal point. Here and in some places elsewhere he uses : instead of / for division. Fewer significant digits are given in the 3rd edn; corresponding to the numbers here are the numbers $48.35,0.55$, $1.3771,3.99,6.11,3.05,1.3374,2.28,8.10,4.10,1.42,2.82,14$.

[37]. In Ref 26 the 1 st 4 is missing from the bottom left entry of the 1 st matrix and the last digit of the bottom right entry of the 3 rd matrix is

2 instead of 1 . The 4 and the 1 are made bold here. In the 3rd edn the matrices are given as $\left(\begin{array}{ccc}1.002 & -3.994 \times 10^{-4} \\ 2.356 & -0.9807\end{array}\right),\left(\begin{array}{cc}0.9807 & -2.686 \times 10^{-3} \\ 49.52 & -0.8841\end{array}\right)$, $\left(\begin{array}{cc}0.9044 & -5.448 \times 10^{-3} \\ 59.94 & 0.7446\end{array}\right)$, respectively, with the leading 4 again missing in the bottom-left entry of the 1st matrix and minus signs inserted in the bottom-right entries of the 1 st and 2 nd matrices.

[38]. $\mathrm{m}$ for metres was missing in the original. This is the distance posterior from the 1 st surface of the eye. In the $3 \mathrm{rd}$ edn the position is given as $1.59 \times 10^{-3}$

[39]. The original has le $p$ p image. The image (or emergent) principal point has reduced position $(c-1) / a=(0.74461-1) / 95.9404$ $=-4.2607 \times 10^{-3} \mathrm{~m}, i e, 4.3224 \mathrm{~mm}$ anterior to the 2 nd surface of the lens of the eye. The index of refraction the vitreous is not given in the appendix. In tableau 1 on p 50 of Ref 26 LG gives it as 1.336. Using this value we find that the actual position is $5.6922 \mathrm{~mm}$ anterior to the $2 \mathrm{nd}$ surface of the crystalline lens of eye. The same table positions the latter surface $7.6 \mathrm{~mm}$ from the 1 st surface of the eye. Hence the emergent principal point is $1.9078 \mathrm{~mm}$ into the eye in agreement with the number given in tableau 1.

[40]. In the original, tableau 1 extends across pp $50 \& 51$ of Ref 26 . The relevant data appear in the column with heading Théorique and subheading non accom. The power and the locations of the principal points are given in the set of rows of the table marked as CEil complet on p 51. Reference to the page number is omitted in the 3rd edn; the table is on pp $74 \& 75$ of that edn and many of the numbers there have fewer significant digits.

[41]. Gauss's coefficients depend on the system and, hence, on the location of the system's entrance and exit planes. The origins are really for longitudinal positions $x$ and $x^{\prime}$ rather than for the coefficients themselves.

[42]. The original has concret.

[43]. One applies Eq (110), starting with the 1st surface of the compensating lens in front of the eye and ending with the posterior surface of the lens of the eye. The resulting matrix gives $a$ and $b$ for use in Eq (109). With the longitudinal position $x$ of the object relative to the 1 st surface of the eye and the height $y$ of the object Eq (109) gives the height $y^{\prime}$ of the image. 
perfect ${ }^{[44]}$; in the case of a remote object the condition of correction is simply written according to (107): ${ }^{[45]}$ $(111)^{[46]} \quad \frac{c}{a}=x^{\prime}$,

$x^{\prime}$ designating the reduced distance from the posterior pole of the crystalline lens to the retina. The use of matrices will greatly simplify the writing of ophthalmic calculations. For example K. N. Ogle ${ }^{[47]}$ used them successfully for studying aniseikonia $(1936)^{[48]}$.

Astigmatic systems. - Let us now consider a system consisting of astigmatic refracting surfaces ${ }^{[49]}$ possessing a common normal (which one takes as axis $\mathrm{O} x$ ) and such that every plane section of one of these refracting surfaces with a plane passing through $\mathrm{O} x$ is symmetric with respect to this axis ${ }^{[50]}$, let us choose any axes $\mathrm{O} y_{1}$ and $\mathrm{O} z_{1}$ forming a trirectangular trihedron with $\mathrm{O} x^{[51]}$.

We know that one of these refracting surfaces is defined by its principal powers $D_{\mathrm{y}}$ and $D_{\mathrm{z}}$ and by the angle $\varphi$ which the principal section $\mathrm{O} y$ makes with plane $x \mathrm{O} y_{1} \cdot{ }^{[52]}$ Instead of these disparate quantities

[44]. supposée au point.

[45]. The condition for compensation with an optical device (such as a lens) in front of the eye would be for $c$ and $a$ of the compound system from the 1st surface of the device to the back surface of the lens of the eye to be such that $c / a$ is the reduced length of the vitreous from the posterior surface of the lens to the retina. One obtains this from Eq (107) by putting $x \rightarrow-\infty$ (a distant object). The result is an indeterminate form which can be resolved by 1 st dividing top and bottom by $x$ to give $x^{\prime}=(c+d / x) /(a+b / x)$ and then neglecting $d / x$ and $b / x$.

[46]. Eq (AII-5) in the 3rd edn.

[47]. Ref 71.

[48]. Instead of '(1936)' the 3rd edn has '[72]' which refers to the Ogle reference on p 398 of Bibliographie.

[49]. dioptres translated here as refracting surfaces.

[50]. Presumably axis $\mathrm{O} x$ is an axis of mirror symmetry of the intersection of the surface and the plane containing $\mathrm{O} x$.

[51]. A plane (dashed in the following sketch) containing $\mathrm{O} x$ intersects the surface with normal along $\mathrm{O} x$ in the curve. The intersection is the dotted line.

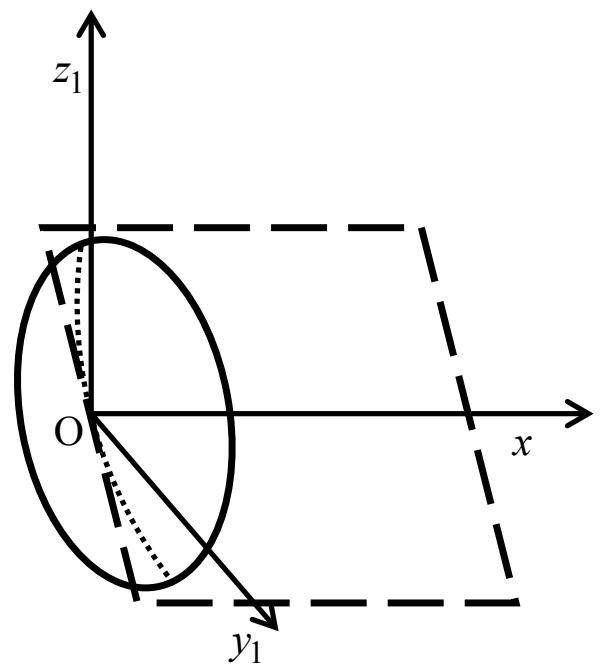

[52]. In the following sketch we are looking along longitudinal axis $\mathrm{O} x . \mathrm{O} y_{1}$ and $\mathrm{O} z_{1}$ are transverse axes, $\mathrm{O} z_{1}$ being perpendicular to $\mathrm{O} y_{1}$ $\mathrm{O} y$ and $\mathrm{O} z$ are the principal meridians of the surface and $D_{y}$ and $D_{z}$ the corresponding principal powers. O $y_{1}$ is the reference transverse axis relative to which angle $\varphi$ is measured; anticlockwise measurements are positive. Although $\mathrm{O} y_{1}$ can be in any direction orthogonal to $\mathrm{O} x$ we shall assume it to be horizontal as in the sketch.

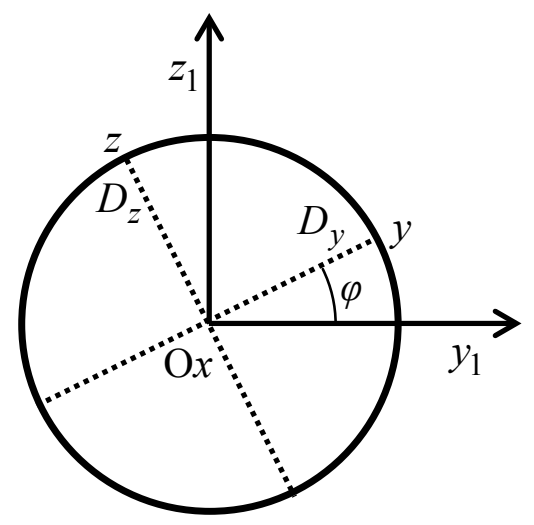


we will introduce three powers ${ }^{[33]}$

$A=\left(D_{y}-D_{z}\right) \sin 2 \varphi$

$B=D_{y} \sin ^{2} \varphi+D_{z} \cos ^{2} \varphi$

$C=D_{y} \cos ^{2} \varphi+D_{z} \sin ^{2} \varphi$.

The system will then be defined by the powers $A$, $B, C$ of each refracting surface, and by the reduced distances $\delta$ which separate them.

Whereas, in Gauss's approximation, a centred system is characterized by three independent parameters ${ }^{[54]}$ [for example, Gauss's four coefficients subject to relation (108)], to the same approximation, the astigmatic system under consideration needs 10 independent parameters ${ }^{[55]}$ : let us consider all the incident rays emitted by the point object; each of them has its direction defined by its three direction cosines $\alpha, \beta, \gamma$ (projections on the coordinate axes of a segment of unit length), these being linked by the relation $\alpha^{2}+\beta^{2}+\gamma^{2}=1$; it suffices for $\beta$ and $\gamma$ to be given to know the ray ${ }^{[56]}$; the corresponding emergent ray ${ }^{[57]}$ will be characterized in the same way by $\beta^{\prime}$ and $\gamma^{\prime}$ and the system establishes a relation ${ }^{[58]}$ $f\left(\beta, \gamma, \beta^{\prime}, \gamma^{\prime}\right)=0$

among these quantities. The approximation similar to that of Gauss ${ }^{[59]}$ consists in retaining, in the development of $f$, only terms with powers up to and including those of second degree, hence 15 coefficients, that is to say one constant, four terms of first order $\left(\beta, \gamma, \beta^{\prime}, \gamma^{\prime}\right)$, four squared terms $\left(\beta^{2}, \gamma^{2}, \beta^{\prime 2}, \gamma^{\prime 2}\right)$ and six rectangular terms $\left(\beta \gamma\right.$, etc) $;{ }^{[60]}$ as the relation remains valid if one

[53]. Comparing these equations with Eqs 30-32 we see that $C=f_{11}$ and $B=f_{22}$. However, because $\sin 2 \varphi=2 \sin \varphi \cos \varphi, A=2 f_{12}$. Hence $C$ and $B$ are the diagonal entries of the dioptric power matrix $\mathbf{F}$ (Eq 29) while $A$ is twice the off-diagonal entries, ie, $\mathbf{F}=\left(\begin{array}{c}C \\ A / 2\end{array} \quad B \quad\right.$. This suggests that the expression for $A$ should be $A=\frac{1}{2}\left(D_{y}-D_{z}\right) \sin 2 \varphi$ and that the omission of the factor $\frac{1}{2}$ is a typographical error. LG does not refer to the dioptric power matrix as such but it appears later as the bottom-left submatrix $\left(\begin{array}{ll}C & A \\ A & B\end{array}\right)$ of the $4 \times 4$ matrix for an astigmatic refracting surface.

[54]. Although often stated, as here, that there are 3 independent parameters, that is not strictly correct. If there were 3 independent parameters one would be able to assign arbitrary values to 3 of the parameters. Assigning the value 0 to any 3 of the parameters violates Eq (108) and shows they are not independent. On the other hand, provided that it exists, the principal matrix logarithm of $\left(\begin{array}{ll}b & d \\ a & c\end{array}\right)$ does have 3 independent parameters
(the off-diagonal entries and 1 of the diagonal entries $)^{44}$.

[55]. A comment similar to Footnote [54] applies here. It is not strictly correct to say that there are 10 independent parameters. Eq (108) is equivalent to saying that $\mathbf{M}$ is symplectic. A symplectic matrix obeys Eq 5. If $\mathbf{M}$ is $4 \times 4$ then the matrix equation $\mathbf{M}^{\mathrm{T}} \mathbf{E} \mathbf{M}=\mathbf{E}$ is equivalent to 6 equations among the 16 entries. The 6 equations are contained within the 3 matrix equations, Eqs 7-9. Provided it exists the principal matrix logarithm of $\mathbf{M}$ has $16-6=10$ independent entries. ${ }^{47}$

[56]. 2 direction cosines uniquely define the incident segment of a ray from the object point.

[57]. The emergent segment of the ray.

[58]. A ray leaving the object point with particular direction cosines $\beta$ and $\gamma$ arrives at the image point with direction cosines $\beta^{\prime}$ and $\gamma^{\prime} ; f$ relates $\beta, \gamma, \beta^{\prime}, \gamma^{\prime}$.

[59]. No reference is given in Refs 26 \& 27. P 328 of Ref 29 gives the reference 'Gauss: Dioptrische Untersuchungen (Göttingen 1840)'. See Ref 69.

[60]. For $\beta$ and $\gamma$ approaching 0 we approximate the function as a sum of terms up to 2 nd degree, ie we write

$$
\begin{array}{rlrl}
A \beta^{2} & +B \gamma^{2}+C \beta^{\prime 2}+D \gamma^{\prime 2} & & \text { (the four squared terms) } \\
& +E \beta \gamma+F \beta \beta^{\prime}+G \beta \gamma^{\prime}+H \gamma \beta^{\prime}+J \gamma \gamma^{\prime}+K \beta^{\prime} \gamma^{\prime} & & \text { (the six rectangular terms) } \\
& +L \beta+M \gamma+N \beta^{\prime}+P \gamma^{\prime} & & \text { (the four terms of first order) } \\
& +Q & & \text { (the constant) } \\
& =0 . &
\end{array}
$$

The 15 coefficients are $A, B, C, D, E, F, G, H, J, K, L, M, N, P, Q$. A ray from an object point through the system obeys this equation. Also the direction cosines become slopes or inclinations. $\beta$ and $\gamma$ become the 2 components $a_{01}$ and $a_{02}$ of the incident vectorial inclination $\mathbf{a}_{0}$ and $\beta^{\prime}$ and $\gamma^{\prime}$ components of the emergent inclination $\mathbf{a}$. 
multiplies these 15 coefficients by the same number only 14 of them are independent; ${ }^{[61]}$ but, further, given the recognized symmetry, nothing changes if $\beta, \gamma$, $\beta^{\prime}$ and $\gamma^{\prime}$ all change sign together ${ }^{[62]}$ which annuls the coefficients of the terms of first order: hence 10 independent parameters.

Just as we defined a centred system by a matrix of second order, we will be able to represent our astigmatic system by a matrix of fourth order, which we will symbolize by $\mathbf{M}$, and write ${ }^{[63]}$

$\mathbf{M}=\left(\begin{array}{llll}e & j & p & m \\ i & f & n & q \\ d & b & g & k \\ a & c & l & h\end{array}\right)$

The 16 terms of this matrix include four powers ${ }^{[64]}$ $(a, b, c, d)$, eight numbers ${ }^{[65]}(e, f, g, h, i, j, k, l)$ and four lengths ${ }^{[66]}(m, n, p, q)$; there exist six relations ${ }^{[67]}$ among these terms, which reduce the number of independent coefficients to 10 .

One can extend the results obtained above for centred systems to astigmatic systems; we will state them without demonstration for which we refer the reader to the reports by T. Smith (1928). ${ }^{[68]}$

1. For a single refracting surface, the origins being coincident with its apex, it becomes ${ }^{[69]}$

$$
\left(\begin{array}{llll}
1 & 0 & 0 & 0 \\
0 & 1 & 0 & 0 \\
C & A & 1 & 0 \\
A & B & 0 & 1
\end{array}\right)=\mathbf{M}
$$

2. Let us displace the origin of the images by a reduced distance $\delta_{1}$. The system $\mathbf{M}_{1}$ becomes ${ }^{[70]}$

$$
\mathbf{M}_{1}\left(\begin{array}{cccc}
1 & 0 & -\delta & 0 \\
0 & 1 & 0 & -\delta \\
0 & 0 & 1 & 0 \\
0 & 0 & 0 & 1
\end{array}\right)=\mathbf{M}
$$

3. In keeping with the same conventions for the origins for the centred system the linking of two systems $\mathbf{M}_{1}$ and $\mathbf{M}_{2}$ is written ${ }^{[71]}$

[61]. The ray also obeys the same equation if all the coefficients are multiplied by a constant. $E g$ if $A$ is not 0 we could multiply all the coefficients by $1 / A$; in effect then $A$ reduces to 1 and we are left with 14 coefficients. The same would apply for any other nonzero coefficient.

[62]. The system has 2-fold rotational symmetry about the optical axis. Consider rays from an object point on the optical axis. Consider in particular a ray whose incident segment has inclinations $\beta$ and $\gamma$; it has emergent inclinations $\beta^{\prime}$ and $\gamma^{\prime}$. Because of the symmetry there is also a ray with incident inclinations $-\beta$ and $-\gamma$ and emergent inclinations $-\beta^{\prime}$ and $-\gamma^{\prime}$. This latter ray must also satisfy the equation in Footnote [60]. Thus the equation must still hold if the signs of all the inclinations are changed which is possible only if $L=M=N=P=0$. 4 more coefficients disappear and we are left with 10.

[63]. As for Gaussian optics (Footnote [26]) $\mathbf{M}$ is $\mathbf{S}^{-1}$. Identifying the entries of $\mathbf{M}$ and $\mathbf{S}^{-1}$ (Eq 41) gives the relationship of LG's 16 scalars to entries of the fundamental properties.

[64]. $d, b, a, c$ are the 4 entries of the transposed power $\mathbf{F}^{\mathrm{T}}$ (see Eq 4). Although $\mathbf{F}$ is symmetric for a thin system (see Eq 31 ) it is not symmetric in general.

[65]. $e, j, i, f$ are entries of $\mathbf{D}^{\mathrm{T}}$ and $g, k, l, h$ entries of $\mathbf{A}^{\mathrm{T}}$.

[66]. $p, m, n, q$ are the entries of $-\mathbf{B}^{\mathrm{T}}$.

[67]. They are the 6 scalar equations contained in matrix Eq 5.

[68]. In the Bibliographie on p 344 there are the 2 references 'T Sмітн, On toric Lenses. [Trans Opt Soc] 29 (1927-1928). p. 71' and 'T. SMITH, The primordial coefficients of Asymmetrical Lenses. Ibid., p 167'. Instead of '(1928)' the 3rd edn has '[76]' and the corresponding entry in the bibliography on p 399 reads 'T. Sмiтн, On toric lenses. Ibid., 29 (1927-1928), 71 et 167'. See Refs 64-68.

[69]. $A, B, C$ are as given above except the missing factor $\frac{1}{2}$ should be inserted in the expression for $A$. See Footnote [53].

[70]. The 3rd edn reads "The system becomes $\mathbf{M}_{1}$ such that ..." This does not seem very clear. Displacing the origins of the images by reduced distance $\delta$ is equivalent to considering the compound system of refracting surface followed by homogeneous gap of reduced width $\delta$. If $\mathbf{M}_{1}$ represents the matrix given above for the refracting surface then the matrix for the compound system becomes

$\mathbf{M}_{1}\left(\begin{array}{cccc}1 & 0 & -\delta & 0 \\ 0 & 1 & 0 & -\delta \\ 0 & 0 & 1 & 0 \\ 0 & 0 & 0 & 1\end{array}\right)=\mathbf{M}$

One is multiplying the inverse transference of an astigmatic refracting surface by that of a homogeneous gap:

$\left(\begin{array}{cccc}1 & 0 & 0 & 0 \\ 0 & 1 & 0 & 0 \\ C & A & 1 & 0 \\ A & B & 0 & 1\end{array}\right)\left(\begin{array}{cccc}1 & 0 & -\delta & 0 \\ 0 & 1 & 0 & -\delta \\ 0 & 0 & 1 & 0 \\ 0 & 0 & 0 & 1\end{array}\right)=\left(\begin{array}{cccc}1 & 0 & -\delta & 0 \\ 0 & 1 & 0 & -\delta \\ C & A & 1-C \delta & -A \delta \\ A & B & -A \delta & 1-B \delta\end{array}\right)=\mathbf{M}$.

Compare Footnote [27]. 


\section{$\mathbf{M}_{1} \mathbf{M}_{2}=\mathbf{M}$.}

4. For a point object at infinity on axis $O x$ the condition for correction of astigmatism is written ${ }^{[72]}$

$$
\frac{l}{a}=\frac{k}{b}=\frac{h}{c}=\frac{g}{d}=x^{\prime}
$$

where $x^{\prime}$ designates, as in equation (111), the reduced abscissa of the image. This correction holds then for points next to the axis.

5. However the image is not like the object; the transverse magnification ${ }^{[74]}$ in particular varies with direction; to correct the defect the supplementary condition $^{[75]}$

$$
(113)^{[76]} \quad c=d \quad(\text { or } g=h)
$$

must be realized.

Application to the eye. - It can happen that the astigmatic eye depends effectively on 10 independent parameters: ${ }^{[77]}$ this happens if the cornea and the two surfaces of the crystalline lens are all three astigmatic with the axes mutually oblique. Even in this case condition (112) can be verified and the eye corrected by an ordinary thin astigmatic lens: ${ }^{[78]}$ this introduces, in effect, four parameters (its three powers $A, B$ and $C$ and its distance to the eye), which will allow the realization of the four equations (112); however, all the possibilities are exhausted and one cannot obtain a magnification independent of the direction. ${ }^{[79]}$ To realize (113) one needs to dispose of one more variable and thus introduce thick correcting systems. ${ }^{[80]}$

Let us consider the simple case in which the cornea presents the only astigmatism; we will realize the correction with a thick glass composed of two toric surfaces separated by a reduced distance $\delta_{2}$, the second face being a distance $\delta_{4}$ in front of the cornea; taking the principal sections (the same for the three surfaces $\left.{ }^{[81]}\right)$ as coordinate planes, conditions

[72]. The compound system from the anterior surface of the optical instrument in front of the eye to the retina must be exit-plane focal, ie Eq 35 holds. Hence the bottom-right $2 \times 2$ submatrix of the inverse transference (Eq 41) must be null. However the inverse transference is

$$
\left(\begin{array}{cccc}
e & j & p & m \\
i & f & n & q \\
d & b & g & k \\
a & c & l & h
\end{array}\right)\left(\begin{array}{cccc}
1 & 0 & -x^{\prime} & 0 \\
0 & 1 & 0 & -x^{\prime} \\
0 & 0 & 1 & 0 \\
0 & 0 & 0 & 1
\end{array}\right)=\left(\begin{array}{cccc}
e & j & p-e x^{\prime} & m-j x^{\prime} \\
i & f & n-i x^{\prime} & q-f x^{\prime} \\
d & b & g-d x^{\prime} & k-b x^{\prime} \\
a & c & l-a x^{\prime} & h-c x^{\prime}
\end{array}\right) .
$$

$\begin{array}{ll}a & c\end{array}$
Hence $g-d x^{\prime}=k-b x^{\prime}=l-a x^{\prime}=h-c x^{\prime}=0$
[73]. (AII-6) in the $3 r d$ edn.

[74]. In the context of the Gaussian optics above 'transverse magnification' means $m=y^{\prime} / y$. The generalization in linear optics would be the matrix $\mathbf{M}$ such that $\mathbf{M y}=\mathbf{y}^{\prime}$, a magnification from a transverse position to a transverse position with $\mathbf{M}$ dimensionless. However this is not what is meant here. Here transverse magnification is a cross-magnification $\mathbf{M}$ from angular position of the object to transverse position of the image: $\mathbf{M a}=\mathbf{y}^{\prime}$. M has the physical dimension of length.

[75]. 2 supplementary conditions are required for sharp images to be free of distortion: conditions (a) and (b) given just after Eq 40. LG's condition ' $c=d$ ' corresponds to the 1 st half of condition (a), equality of the diagonal elements of the dioptric power $\mathbf{F}$ or the rotation matrix of Eq 38. It omits the 2nd part of condition (a) and the whole of condition (b). It follows that his condition is neither necessary nor sufficient. If $c=d$ then for no distortion it must also be true that $a=-b$ (corresponding to the off-diagonal elements of Eq 38). However it is also possible for image size to be independent of orientation of the object if $c=-d$ and then it must also be true that $a=b$ (corresponding to Eq 39). The necessary and sufficient condition for image size to be independent of object direction then is that $\left(\begin{array}{ll}c & a\end{array}\right)= \pm\left(\begin{array}{ll}d & -b\end{array}\right)$ or $\left(\begin{array}{ll}g & l\end{array}\right)= \pm(h-k)$. However, if one limits consideration to the naked eye and the eye compensated by means of conventional optical devices including spectacle and contact lenses then it is safe to disregard condition (b). Furthermore the off-diagonal elements of $\mathbf{F}$ in these cases are usually close to 0 so that one expects the 2 nd part of condition (a), namely that $a=-b$, to be nearly satisfied anyway. It follows that LG's condition can be regarded as necessary and sufficient for most practical applications in optometry.

[76]. Eq (AII-7) in the 3rd edn.

[77]. This is true of eyes as of optical systems in general. But see Footnote [55].

[78]. Eq 46 gives the divergence or power of the thin compensating lens.

[79]. This is usually true. However image size is independent of object orientation in the case of an eye for which there exists a scalar $\zeta_{1}$ such that $\mathbf{A} \zeta_{1}+\mathbf{B}$ is a scalar multiple of a rotation (or reflection) matrix, a result that follows from application of Eq 17 to the system of lens and eye (Eqs $44 \& 45)$. An obvious example is an eye with no astigmatic refracting surfaces.

[80]. Application of Eqs $35 \& 40$ to the compound system of eye and optical instrument (Eq 48) shows that the eye and instrument must satisfy both Eqs $49 \& 50$.

[81]. Because the principal meridians of the 3 surfaces are matching linear optics is unnecessary; Gaussian optics can be used twice, once in each principal meridional plane. Using linear optics here we choose the reference meridian $\mathrm{O} y_{1}$ to lie in a principal meridional plane $\mathrm{O} y$. Hence $\varphi=0$. 
(112) and (113) reduce to ${ }^{[82]}$

$B_{1}\left[1+\left(\delta_{2}+\delta_{4}\right) R_{\mathrm{y}}\right\rfloor+B_{3}\left(1+\delta_{4} R_{\mathrm{y}}\right)-B_{1} B_{3}\left(\delta_{2}+\delta_{2} \delta_{4} R_{\mathrm{y}}\right)=R_{\mathrm{y}}$ $C_{1}\left[1+\left(\delta_{2}+\delta_{4}\right) R_{\mathrm{z}}\right]+C_{3}\left(1+\delta_{4} R_{\mathrm{z}}\right)-C_{1} C_{3}\left(\delta_{2}+\delta_{2} \delta_{4} R_{\mathrm{z}}\right)=R_{\mathrm{z}}$ $\left(C_{1}-B_{1}\right)\left(\delta_{2}+\delta_{4}\right)+\left(C_{3}-B_{3}\right) \delta_{4}-\left(C_{1} C_{3}-B_{1} B_{3}\right) \delta_{2} \delta_{4}=0$

where $R_{\mathrm{y}}$ and $R_{\mathrm{z}}$ designate the ametropias measured at the corneal apex. There ${ }^{[83]}$ is an infinity of solutions, which allows a supplementary condition to be given.

For example, for $\delta_{2}=4 \mathrm{~mm}, \delta_{4}=12 \mathrm{~mm}, R_{\mathrm{y}}=-7$ dt and $R_{\mathrm{z}}=-5 \mathrm{dt}$, one can adopt the following values: $B_{1}=+6.38 \quad \mathrm{dt}^{[84]} \quad B_{3}=-14.19 \quad \mathrm{dt}^{[85]} \quad C_{1}=0$ $C_{3}=-5.32 \mathrm{dt}$.

However, as long as one imposes a reasonable limit on the thickness of the correcting glass, it will only be possible in this way to correct those astigmatisms that barely exceed $2 \mathrm{dt} ;{ }^{[86]}$ and in this case the deformations which result from variation of the transverse magnification remain sufficiently weak for it not to be necessary to calculate it. ${ }^{[87]}$

[82]. The compensating system in front of the eye has transference given by Eq 34. For a sharp image it must satisfy Eq 49 or, equivalently, Eq 51. We substitute for $\mathbf{A}_{\mathrm{C}}$ and $\mathbf{C}_{\mathrm{C}}$ from Eq 34 into Eq 49 and make use of Eq 4. After a little rearrangement we arrive at the condition for a sharp image:

$\left(\mathbf{I}+\left(\delta_{2}+\delta_{4}\right) \mathbf{F}_{0}\right) \mathbf{F}_{1}+\left(\mathbf{I}+\delta_{4} \mathbf{F}_{0}\right) \mathbf{F}_{3}-\delta_{2}\left(\mathbf{I}+\delta_{4} \mathbf{F}_{0}\right) \mathbf{F}_{3} \mathbf{F}_{1}=\mathbf{F}_{0}$

This holds in general. We now apply it to the particular example. Because $\varphi=0$ we have for the 1st surface of the lens $\mathbf{F}_{1}=\left(\begin{array}{cc}C_{1} & 0 \\ 0 & B_{1}\end{array}\right)$ and similarly for the 2nd surface. Also $\mathbf{F}_{0}=\left(\begin{array}{cc}R_{y} & 0 \\ 0 & R_{z}\end{array}\right)$ where $R_{y}$ is the corneal-plane refractive compensation in the reference meridian and $R_{z}$ in the meridian orthogonal to it. All the matrices in Eq 57 are diagonal. Multiplying out we obtain the 2 scalar equations

$\left\lfloor 1+\left(\delta_{2}+\delta_{4}\right) R_{y}\right\rfloor C_{1}+\left(1+\delta_{4} R_{y}\right) C_{3}-\delta_{2}\left(1+\delta_{4} R_{y}\right) C_{1} C_{3}=R_{y}$

$\left[1+\left(\delta_{2}+\delta_{4}\right) R_{z}\right] B_{1}+\left(1+\delta_{4} R_{z}\right) B_{3}-\delta_{2}\left(1+\delta_{4} R_{z}\right) B_{1} B_{3}=R_{z}$

which are identical to the 1 st 2 of LG's 3 equations except that $y$ and $z$ are interchanged. His equations are obtained if we choose O $y_{1}$ to coincide with $\mathrm{O} z$ instead of $\mathrm{O} y$ in which case $\varphi=90^{\circ}$. For an undistorted image Eq 55 must also be satisfied. The transference of the cornea is $\mathbf{S}_{\mathrm{K}}=\left(\begin{array}{cc}\mathbf{I} & \mathbf{O} \\ -\mathbf{F}_{\mathrm{K}} & \mathbf{I}\end{array}\right)$ where $\mathbf{F}_{\mathrm{K}}=\left(\begin{array}{cc}C_{\mathrm{K}} & 0 \\ 0 & B_{\mathrm{K}}\end{array}\right)$. Because there are no astigmatic elements in the rest of the eye the fundamental properties of the rest of the eye are scalar matrices. Thus we can write $\mathbf{S}_{\mathrm{R}}=\left(\begin{array}{cc}A_{\mathrm{R}} \mathbf{I} & B_{\mathrm{R}} \mathbf{I} \\ C_{\mathrm{R}} \mathbf{I} & D_{\mathrm{R}} \mathbf{I}\end{array}\right)$ for the transference of the rest of the eye. Hence the transference of the eye is

$\mathbf{S}=\left(\begin{array}{cc}A_{\mathrm{R}} \mathbf{I} & B_{\mathrm{R}} \mathbf{I} \\ C_{\mathrm{R}} \mathbf{I} & D_{\mathrm{R}} \mathbf{I}\end{array}\right)\left(\begin{array}{cc}\mathbf{I} & \mathbf{O} \\ -\mathbf{F}_{\mathrm{K}} & \mathbf{I}\end{array}\right)=\left(\begin{array}{cc}A_{\mathrm{R}} \mathbf{I}-B_{\mathrm{R}} \mathbf{F}_{\mathrm{K}} & B_{\mathrm{R}} \mathbf{I} \\ C_{\mathrm{R}} \mathbf{I}-D_{\mathrm{R}} \mathbf{F}_{\mathrm{K}} & D_{\mathrm{R}} \mathbf{I}\end{array}\right)$

and so $\mathbf{B}=B_{\mathrm{R}} \mathbf{I}$. Hence Eq 55 becomes

$\mathbf{I}-\delta_{2} \mathbf{F}_{1}-\delta_{4}\left(\mathbf{F}_{3}\left(\mathbf{I}-\delta_{2} \mathbf{F}_{1}\right)+\mathbf{F}_{1}\right)=-m B_{\mathbf{R}} \mathbf{R}$.

Again the matrix on the left is diagonal, the diagonal entries being

$1-\delta_{2} C_{1}-\delta_{4}\left(C_{3}\left(1-\delta_{2} C_{1}\right)+C_{1}\right)$

$1-\delta_{2} B_{1}-\delta_{4}\left(B_{3}\left(1-\delta_{2} B_{1}\right)+B_{1}\right)$.

Because the off-diagonal entries are 0 Eq 55 can be satisfied in 2 distinct ways: $\theta=0^{\circ}$, in which case $\mathbf{R}=\mathbf{R}_{0^{\circ}}=\mathbf{I}$, or $\theta=45^{\circ}$, in which case $\mathbf{R}=\overline{\mathbf{R}}_{45^{\circ}}$. In the 1 st case the diagonal entries are equal; their difference is 0 from which we obtain the 3 rd of LG's equations. In the 2 nd case their sum is 0 which results in

$\left(C_{1}+B_{1}\right)\left(\delta_{2}+\delta_{4}\right)+\left(C_{3}+B_{3}\right) \delta_{4}-\left(C_{1} C_{3}+B_{1} B_{3}\right) \delta_{2} \delta_{4}=2$.

The 2 cases correspond to conditions (a) and (b) respectively (see Condition for sharp undistorted images). Although a theoretical alternative to LG's 3rd equation this equation leads to absurd results in ordinary applications when used instead of his equation. Eg if we take LG's values for $\delta_{2}, \delta_{4}, C_{1}$ and use this equation instead of his 3 rd equation we obtain the powers $B_{1}=493.63 \mathrm{D}, C_{3}=-5.32 \mathrm{D}, B_{3}=498.91 \mathrm{D}$. Alternatively if $\delta_{2}=\delta_{4}=1 \mathrm{~m}, C_{1}=2 \mathrm{D}$ we obtain $B_{1}=-0.5 \mathrm{D}, C_{3}=3.25 \mathrm{D}, B_{3}=1.5 \mathrm{D}$. Apparently if widths are reasonable powers are ridiculous and if powers are reasonable widths are ridiculous. This serves to illustrate the fact that condition (b) can be disregarded in practice in ordinary optometric applications.

[83]. There is a typographical error in the original (corrected in the 3rd edn): 'I y a' should read 'Il y a'.

[84]. The original has 5 instead of the correct 8 shown bold here.

[85]. The original has 6 instead of the correct 9 shown bold here.

[86]. By astigmatism LG evidently means $\left|R_{\mathrm{y}}-R_{\mathrm{z}}\right|$.

[87]. In the 3rd edn LG adds a 1-sentence paragraph: "For these applications of matrix arithmetic to ophthalmic optics one can consult the paper by Miss Bourdy [55]." On p 398 under Bibliographie Ref 27 reads [55]'C. Bourdy, Calcul matriciel et optique paraxiale. Rev. d'Opt., 41 (1962) 295.' See Ref 72. 
EXERCISES [p 329]

[p 332]

EXERCISE 43 (Appendix II). - By means of Gauss's coefficients establish the formulae associated with Gullstrand.

EXERCISE 44 (Appendix II). - The same problem in frontal notation.

\section{SOLUTIONS TO THE EXERCISES [p 333]}

[p 341]

43. - If the component systems are referred to their principal points, expression (110) provides us with the resulting matrix

$\left(\begin{array}{cc}1 & 0 \\ D_{1} & 1\end{array}\right)\left(\begin{array}{cc}1 & -\delta \\ 0 & 1\end{array}\right)\left(\begin{array}{cc}1 & 0 \\ D_{2} & 1\end{array}\right)=\left(\begin{array}{ll}b & d \\ a & c\end{array}\right)$,

which gives

$a=D_{1}+D_{2}-\delta D_{1} D_{2}$,

$b=1-\delta D_{2}$,

$c=1-\delta D_{1}$,

$d=-\delta$.

We have seen that the resulting power was $a$, and that the reduced abscissas of the principal points are written $(1-b) / a$ and $(c-1) / a$. Whence Gullstrand's formulae. ${ }^{[88]}$

44. - In designating the abscissa in the first system by $x_{0}$ from the resulting object origin one obtains ${ }^{[89]}$

$$
\begin{aligned}
& \left(\begin{array}{cc}
g_{1}^{\prime} & 0 \\
D_{1}^{\prime} / g_{1}^{\prime} & 1 / g_{1}^{\prime}
\end{array}\right)\left(\begin{array}{cc}
1 & -\xi \\
0 & 1
\end{array}\right)\left(\begin{array}{cc}
g_{2}^{\prime} & 0 \\
D_{2}^{\prime} / g_{2}^{\prime} & 1 / g_{2}^{\prime}
\end{array}\right) \\
& =\left(\begin{array}{cc}
1 & -x_{0} \\
0 & 1
\end{array}\right)\left(\begin{array}{cc}
g^{\prime} & 0 \\
D^{\prime} / g^{\prime} & 1 / g^{\prime}
\end{array}\right) .
\end{aligned}
$$

Whence the classical formulae by identification (see exercise 22) ${ }^{[90]}$.

\section{References}

1. Langenbucher A, Reese S, Sauer T, Seitz B. Matrix-based calculation scheme for toric intraocular lenses. Ophthal Physiol Opt 200424 511-519.

2. Langenbucher A, Reese S, Seitz B. Impact of decentration of astigmatic intra-ocular lenses on the residual refraction after cataract surgery. Ophthal Physiol Opt 200525 568-575.

3. Langenbucher A, Szentmáry N, Speck A, Seitz B, Eppig T. calculation of power and field of view of keratoprostheses. Ophthal Physiol Opt 201333 412-419.

4. Ale JB, Manns F, Ho A. Evaluation of the performance of accommodating IOLs using a paraxial optics analysis. Ophthal Physiol Opt 201030 132-142.

5. Ale JB, Manns F, Ho A. Magnifications of single and dual element accommodation intraocular lenses: paraxial optics analysis. Ophthal Physiol Opt 2011 31 7-16.

6. Espinós M, Micó V. Lateral magnification matrix from the dioptric power matrix formalism in the paraxial case. Ophthal Physiol Opt 201333 467-481.

7. MacKenzie GE, Harris WF. Determining the power of a thin toric intraocular lens in an astigmatic eye. Optom Vis Sci 200279 667-671.

8. MacKenzie GE, Harris WF, van Gool RD. Comparison of first-order schematic eyes in 20-dimensional inner-product

[88]. On p 343 of his Bibligraphie LG gives the reference 'A. Gullstrand, Einführung in die Methoden de Dioptrik des Auges des Menschen. (Leipzig, 1911)'. The formula for $a$ is the formula often referred to as Gullstrand's formula. Notice the order of multiplication $D_{1} D_{2}$; it is correct here (in Gaussian optics) but must be reversed to $\mathbf{D}_{2} \mathbf{D}_{1}$ when the formula is generalized to linear

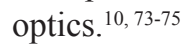

[89]. Multiplication results in

$\left(\begin{array}{cc}g_{1}^{\prime}\left(g_{2}^{\prime}-\xi D_{2}^{\prime} / g_{2}^{\prime}\right) & -g_{1}^{\prime} \xi / g_{2}^{\prime} \\ \left(g_{2}^{\prime} D_{1}^{\prime}+\left(1-\xi D_{1}^{\prime}\right) D_{2}^{\prime} / g_{2}^{\prime}\right) / g_{1}^{\prime} & \left(1-\xi D_{1}^{\prime}\right) /\left(g_{1}^{\prime} g_{2}^{\prime}\right)\end{array}\right)=\left(\begin{array}{cc}g^{\prime}-x_{0} D^{\prime} / g^{\prime} & -x_{0} / g^{\prime} \\ D^{\prime} / g^{\prime} & 1 / g^{\prime}\end{array}\right)$.

Equating the bottom-right entries and rearranging we obtain the shape factor

$g^{\prime}=\frac{g_{1}^{\prime} g_{2}^{\prime}}{1-D_{1}^{\prime} \xi}$,

which is the equation near the bottom of p 108 of LG and Eq 10.5 of LG\&EH. From the bottom-left entries and Eq 65 we obtain the back-vertex power (frontal rear power in LG\&EH) $D^{\prime}=\frac{g_{2}^{\prime 2} D_{1}^{\prime}}{1-D_{1}^{\prime} \xi}+D_{2}^{\prime}$, Eq 58 on p 108 of LG and Eq 10.4 of LG\&EH.

[90]. Ex 22 (on p 330 of LG) reads: 'By means of homography examine the combination of two centred systems in frontal notation.' The solution is on $\mathrm{p} 337$ of LG. 
space. S Afr Optom $2004 \mathbf{6 3} 103-108$.

9. Acosta E, Blendowske R. Paraxial optics of astigmatic systems: relations between the wavefront and the ray picture approaches. Optom Vis Sci 2007 84, E72-E78.

10. Diepes H, Blendowske R. Optik und Technik der Brille. Heidelberg: Optische Fachveröffentlichung 2002 477-511.

11. Becken W, Altheimer H, Esser G, Mueller W, Uttenweiler D. Optical magnification matrix: near objects in the paraxial case. Optom Vis Sci 200885 E581-E592.

12. Becken W, Altheimer H, Esser G, Mueller W, Uttenweiler D. Optical magnification matrix: near objects and strongly oblique incidence. Optom Vis Sci 200885 E593-E604.

13. García M, González C, Pascual I. New matrix formulation of spectacle magnification using pupil magnification. I. High myopia corrected with ophthalmic lenses. Ophthal Physiol Opt 199515 195-205.

14. García M, González C, Pascual I. A new matrix formulation of spectacle magnification using pupil magnification. II and III. High myopia corrected with contact lenses and intraocular lenses. Ophthal Physiol Opt 199616 498-506.

15. Harris WF. Optical effects of ocular surgery including anterior segment surgery. J Cataract Refract Surgery 2001 27 95-106.

16. Harris WF. Achromatic axes and their linear optics. Vis Res 201258 1-9.

17. Harris WF. Chief nodal axes of a heterocentric astigmatic eye and the Thibos-Bradley achromatic axis. Vis Res 2012 73 40-45.

18. Harris WF, van Gool RDHM, Evans T. Line of sight of a heterocentric astigmatic eye. Ophthal Physiol Opt 201333 57-66.

19. Smith G, Atchison DA. The Eye and Visual Optical Instruments. Cambridge: Cambridge University Press 1997 752-772.

20. Long WF. A matrix formalism for decentration problems. Am J Optom Physiol Opt 197653 27-33.

21. Colliac JP. Matrix formula for intraocular lens power calculation. Invest Ophthalmol Vis Sci 199031 374-381.

22. Keating MP. A system matrix for astigmatic optical systems: I. Introduction and dioptric power relations. Am J Optom Physiol Opt 198158 810-819.

23. Keating MP. A system matrix for astigmatic optical systems: II. Corrected systems including an astigmatic eye. $\mathrm{Am} \mathrm{J}$ Optom Physiol Opt 198158 919-929.

24. Blendowske R. Hans-Heinrich Fick: early contributions to the theory of astigmatic systems. S Afr Optom 200362105 110 .

25. Fick HH. Fortschrittliche Rechnungsarten in der Augenoptik. 11. Folge. Der Augenoptiker 1972 (12) 60-63.

26. Le Grand Y. Optique Physiologique: Tome Premier: La Dioptrique de l'Eil et Sa Correction. Paris: Éditions de la "Revue d'Optique" 1945.

27. Le Grand Y. Optique Physiologique. Tome Premier. La Dioptrique de l'CEil et Sa Correction (3rd ed). Paris: Masson 1964 374-381. [WFH's copy gives the publisher the same as that for the first edition (see Ref 26) and carries the date 1965 on the cover and 1964 on the title page. However the publisher's name has been pasted over with the new name
(Masson).]

28. Le Grand Y. Preface to the English edition. Page vii of Ref 29.

29. Le Grand Y, El Hage SG. Physiological Optics. Berlin: Springer 1980.

30. Guillemin V, Sternberg S. Symplectic Techniques in Physics. Cambridge: Cambridge University Press 19847 et seq.

31. Ditteon R. Modern Geometric Optics. New York: Wiley 1998176.

32. Klein MV, Furtak TE. Optics (2nd ed). New York: Wiley 1986153.

33. Blaker JW. Geometric Optics: The Matrix Theory. New York: Dekker 197129.

34. Lipson SG, Lipson H, Tannhauser DS. Optical Physics (3rd ed). Cambridge: Cambridge University Press 199553.

35. Brouwer W. Matrix Methods in Optical Instrument Design. New York: Benjamin 196448.

36. Das P. Lasers and Optical Engineering. New York: Springer 199113.

37. Torre A. Linear Ray and Wave Optics in Phase Space. Amsterdam: Elsevier 2005 12, 37-40, 60, 155, 179.

38. Gerrard A, Burch JM. Introduction to Matrix Methods in Optics. London: Wiley 1975 26, 28.

39. Harris WF. Dioptric power: its nature and its representation in three- and four-dimensional space. Optom Vis Sci 1997 74 349-366.

40. Harris WF. A unified paraxial approach to astigmatic optics. Optom Vis Sci 199976 480-499.

41. Harris WF. The four fundamental properties of Gaussian optical systems including the eye. S Afr Optom 199958 6979.

42. Hall BC. Lie Groups, Lie Algebras, and Representations: an Elementary Introduction. New York: Springer 20038.

43. McDuff D, Salamon D. Introduction to Symplectic Topology (2nd ed). Oxford: Clarendon Press 1998 19, 20.

44. Bernstein DS. Matrix Mathematics: Theory, Facts, and Formulas (2nd ed). Princeton: Princeton University Press 2009183.

45. Fassbender H. Symplectic Methods for the Symplectic Eigenproblem. New York: Kluwer 20102.

46. Higham NJ. Functions of Matrices: Theory and Computation. Philadelphia: SIAM 2008323.

47. Harris WF. Symplecticity and relationships among the fundamental properties in linear optics. S Afr Optom 2010 69 3-13.

48. Serre D. Matrices: Theory and Applications. New York: Springer 2002 121-128.

49. Kauderer M. Symplectic Matrices: First Order Systems and Special Relativity. Singapore: World Scientific 1994 8, 16.

50. Harris WF. Paraxial ray tracing through noncoaxial astigmatic optical systems, and a 5x5 augmented system matrix. Optom Vis Sci 199471 282-285.

51. Harris WF, Cardoso JR. The exponential-mean-logtransference as a possible representation of the optical character of an average eye. Ophthal Physiol Opt 200626 380-383.

52. Cardoso JR, Harris WF. Transformations of ray transferences of optical systems to augmented Hamiltonian matrices and 
the problem of the average system. S Afr Optom $2007 \mathbf{6 6}$ 56-61.

53. Harris WF. Quantitative analysis of transformed transferences of optical systems in a space of augmented Hamiltonian matrices. S Afr Optom 200766 62-67.

54. Harris WF. Interconverting the matrix and principalmeridional representations of dioptric power and reduced vergence. Ophthal Physiol Opt 200020 494-500.

55. Schur I. Über Potenzreihen, die im Innern des Einheitkreises beschränkt sind. J Reine Angew Math 1917147 205-232.

56. Zhang F. Editor of: The Schur Complement and Its Applications. Springer, New York, 2005.

57. Dopico FM, Johnson CJ. Complementary bases in symplectic matrices and a proof that their determinant is one. Linear Algebra Its Appl 2006419 772-778.

58. Harris WF. Cardinal points and generalizations. Ophthal Physiol Opt 201030 391-401.

59. Harris WF. Conditions in linear optics for sharp and undistorted retinal images, including Le Grand's conditions for distant objects. Vis Res 201393 88-94.

60. Herzberger M. On the fundamental optical invariant, the optical tetrality principle, and on the new development of Gaussian optics based on this law. J Opt Soc Am 1935 25 295-304.

61. Herzberger M. First-order laws in asymmetrical optical systems. Part I. The image of a given congruence: fundamental conceptions. J Opt Soc Am 193626 354-359.

62. Herzberger M. First-order laws in asymmetrical optical systems. II. The image congruences belonging to the rays emerging from a point in object and image space; fundamental forms. J Opt Soc Am 193626 389-406.

63. Smith T. The back vertex power of a combination of lenses. Trans Opt Soc 192426 31-37.

64. Smith T. On toric lenses. Trans Opt Soc 192729 71-87.

65. Smith T. Canonical forms in the theory of asymmetrical optical systems. Trans Opt Soc 192729 88-98.

66. Smith T. The primordial coefficients of asymmetrical lenses. Trans Opt Soc 192829 167-178.

67. Smith T. The need for reform in the teaching of optics. Proc Phys Soc Lond 192941 258-269.

68. Smith T. On tracing rays through an optical system. Proc Phys Soc Lond 194557 286-293.

69. Gauss CG. Dioptrische Untersuchungen. Abhandlungen der Königlichen Gesellschaft der Wissenschaften in Göttingen (übergeben 1840 December 10) 1843 1-34.

70. Pendlebury C. Lenses and Systems of Lenses treated after the Manner of Gauss, Cambridge: Deighton, Bell and Co, 188455.

71. Ogle KN. The correction of aniseikonia with ophthalmic lenses. J Opt Soc Am 193626 323-337.

72. Bourdy C. Calcul matriciel at optique paraxiale: application à l'optique ophthalmique. Rev d'Opt 196241 295-308.

73. Keating MP. Advantages of a block matrix formulation for an astigmatic system. Am J Optom Physiol Opt 591982 851857.

74. Keating MP. A matrix formulation of spectacle magnification. Ophthal Physiol Opt 19822 145-158.

75. Fick HH. Fortschrittliche Rechnungsarten in der Augenoptik. 22. Folge und Schluß. Der Augenoptiker 1974 (6) 49-51. 